\title{
7. DIATOM BIOSTRATIGRAPHY OF EQUATORIAL INDIAN OCEAN SITE 758 ${ }^{1}$
}

\author{
Elisabeth Fourtanier ${ }^{2}$
}

\begin{abstract}
Diatoms occur at Site 768 in the equatorial Indian Ocean in three intervals: (1) Quaternary to upper Pliocene, (2) lower Miocene to upper Oligocene, and (3) upper Campanian. The diatom assemblages are documented for each of these intervals. Similarities between the diatom flora in the equatorial Pacific and at Site 758 allow diatom zonations established in the equatorial Pacific to be used at Site 758, and the presence of a hiatus of about 3-m.y. duration at the Miocene/Oligocene boundary is demonstrated. Upper Pliocene to Quaternary diatom datums levels calibrated to magnetostratigraphy are compared between Site 758, the equatorial Pacific Ocean, and the North Atlantic Ocean. Diachroneity is shown for some of the events. One new taxa, Craspedodiscus elegans var. asymmetricus, n. var., is described, and one new combination, Rossiella magna (Lohman) n. comb., is proposed.
\end{abstract}

\section{INTRODUCTION}

\section{Geographical and Geological Setting}

Ocean Drilling Program (ODP) Site 758 lies along the crest of Ninetyeast Ridge, at the approximate position of $5^{\circ} 23^{\prime} \mathrm{N}, 90^{\circ}$ $21^{\prime} \mathrm{E}$, in a water depth of $2924 \mathrm{~m}$ (Fig. 1).

Ninetyeast Ridge is interpreted as the trace of the Kerguelen/Ninetyeast hotspot on the Indian plate prior to rifting along the incipient Southeast Indian Ridge, which separated from the Indian plate in the middle Eocene (Pierce, Weissel, et al., 1989). As a result of the northward movement of Ninetyeast Ridge, sediments at Site 758 were deposited at different latitudes; thus the record at this site represents temperate environments during the Campanian becoming tropical in the Maestrichtian (Leg 121 Shipboard Scientific Party, 1988). Predicted paleolatitudes for Site 758 (Peirce, Weissel, et al., 1989, fig. 16, p. 21) indicate that the site was situated on the equator in the middle Miocene, at about $5^{\circ} \mathrm{S}$ around the Oligocene/Miocene boundary, and at temperate latitudes during the Campanian.

\section{Diatoms in the Sediments of Site 758}

Sediments recovered at this site range from Quaternary to Campanian. The sedimentary record is nearly complete for the Neogene; however, several disconformities are present in the Paleogene and the lower Oligocene, most of the Eocene, and the lower Paleocene are missing (Peirce, Weissel, et al., 1989). Diatoms at Site 758 were recovered from three distinctive intervals: (1) the Quaternary and upper Pliocene; (2) the lower Miocene and upper Oligocene; and (3) a short interval in the Campanian. Dissolution-resistant diatoms are rare between the lower Pliocene and the middle Miocene (Peirce, Weissel, et al., 1989). Sediments of Eocene, Paleocene, and Maestrichtian ages are barren of diatoms.

\section{Late Neogene}

In contrast to the equatorial Pacific, where late Neogene diatoms have been actively studied (for references see Barron,

${ }^{1}$ Weissel, J., Peirce, J., Taylor, E., Alt, J., et al., 1991. Proc. ODP, Sci. Results, 121: College Station, TX (Ocean Drilling Program).

${ }^{2}$ Lab. d'Hydrologie et Géochimie Isotopique, Université Paris-Sud, Orsay, France. (Mailing address: U.S. Geological Survey, Branch of Paleontology and Stratigraphy, MS 915, 345 Middlefield Road, Menlo Park, CA 94025, U.S.A.)
1985 b , 1985c), relatively few diatom studies have been completed on late Neogene to Quaternary diatoms from the tropical Indian Ocean. These include the works of Schrader (1974, DSDP Leg 24), Kazarina (1975), Jousé and Kazarina (1975, DSDP Leg 27), Burckle (1989), and Mikkelsen (1990, Leg 115). Furthermore, estimation of absolute ages of Neogene diatom datums in the equatorial Pacific has been possible by correlation with magnetostratigraphy (Burckle, 1972; Barron, 1985b, in press), whereas similar studies for the equatorial Indian Ocean are very limited (Burckle and Opdyke, 1977). As magnetostratigraphy is available for the upper Neogene of Site 758, study of upper Pliocene to Quaternary diatoms at this site is an excellent opportunity to determine the ranges of diatom taxa in the equatorial Indian Ocean and to establish comparisons with the equatorial Pacific Ocean.

\section{Lower Miocene and Upper Oligocene}

Studies of low-latitude upper Oligocene and lower Miocene diatom sequences are relatively uncommon. The most significant studies have been completed in the low-latitudes areas of the Pacific Ocean (e.g., Jousé, 1973; Barron, 1983, 1985b; Kim and Barron, 1986; Oreshkhina and Radionova, 1987; Radionova, 1987) and in the low-latitude Atlantic Ocean (Schrader, 1978; Fenner, 1982, 1984).

As upper Oligocene and lower Miocene stratigraphic sequences of diatom assemblages have not been documented until now from the tropical Indian Ocean, Site 758 represents, therefore, a unique contribution to the knowledge of spacial and temporal distribution of diatoms in the low latitudes during the late Oligocene-early Miocene interval.

\section{Cretaceous}

The most continuous sequences of Upper Cretaceous diatomaceous sediments are known from the USSR (e.g., Strelnikova, 1974, documented Upper Cretaceous diatom sequences from western Siberia and the eastern slope of the eastern Ural mountains). Despite the relatively poor preservation of the assemblages and the short range of the diatomaceous interval, the Campanian diatoms at Site 758 are of interest as the occurrence of Cretaceous diatom sequences is rather uncommon in the deep-sea record. This includes mostly DSDP Site 275 in the South Pacific (Hajos and Stradner, 1975), DSDP Site 216 in the equatorial Indian Ocean (Bukry, 1974), and Canadian Expedition to Study the Alpha Ridge (CESAR 6) core in the Arctic Ocean (Barron, 1985a). 


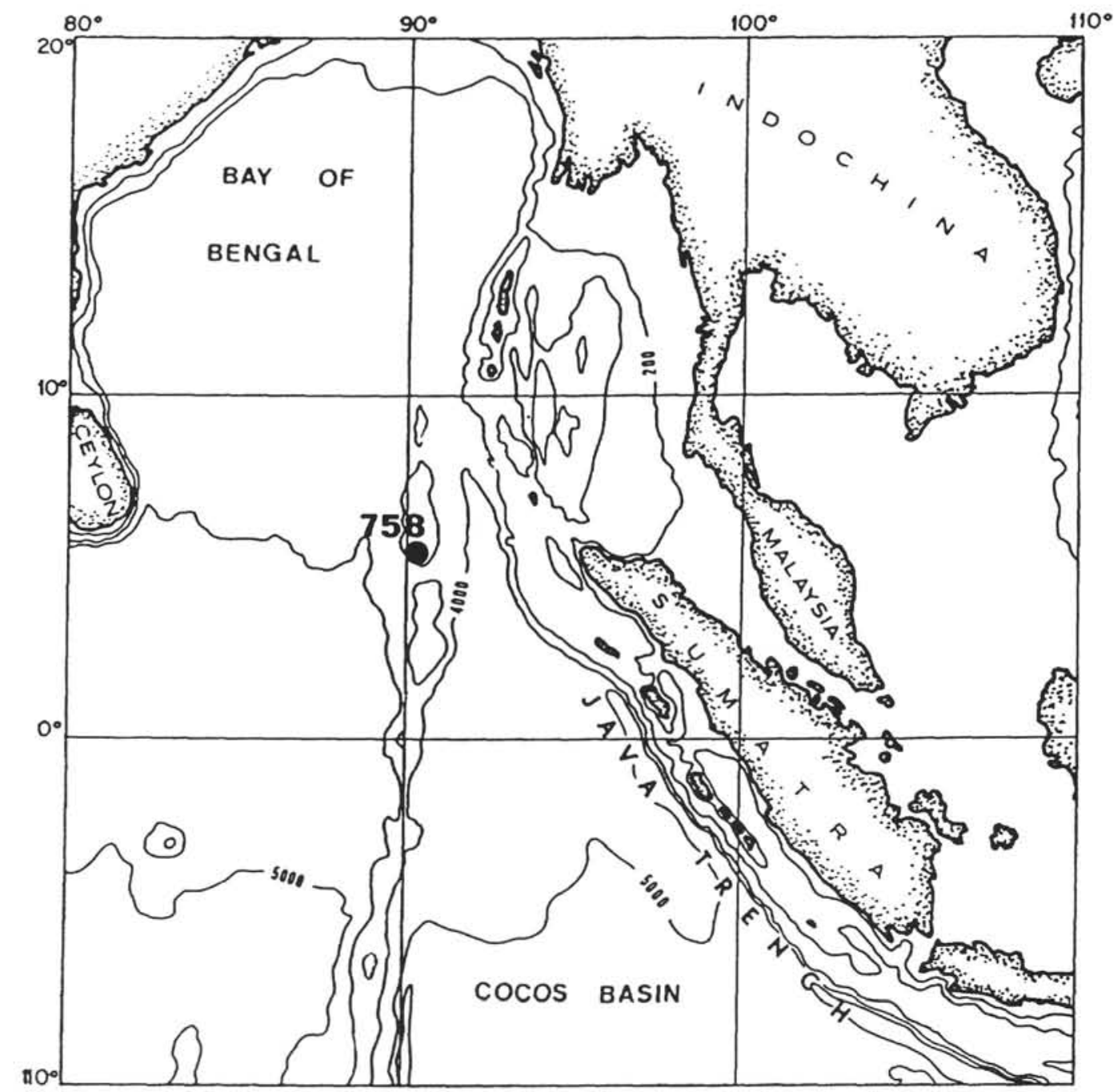

Figure 1. Location of Site 758 at the northern end of Ninetyeast Ridge.

\section{Objectives}

This chapter documents the diatom assemblages recovered from Hole 758A. The stratigraphic ranges of the upper Oligocene and younger diatoms are compared with those from the equatorial Pacific and North Atlantic Oceans. In addition, the utility in the tropical Indian Ocean of late Paleogene and Neogene diatom biostratigraphies established in the equatorial Pacific is discussed.

\section{METHODS}

Samples ( 1 to $3 \mathrm{~g}$ ) were boiled with $20 \%$ hydrochloric acid $(30$ $\mathrm{cm}^{3}$ ) and $30 \%$ hydrogen peroxide $\left(15 \mathrm{~cm}^{3}\right)$ to remove calcium carbonate and organic matter. The residues prepared aboard the JOIDES Resolution were rinsed by centrifuging the suspension ( $75 \%$ full speed for $5 \mathrm{~min}$ ), decanting off the liquid, and washing by addition of distilled water (this cycle was repeated three times). The residues prepared onshore were rinsed by washing in distilled water in a $250 \mathrm{~mL}$ beaker, settling for a minimum of $8 \mathrm{hr}$, and decanting the liquid away (this cycle was repeated three or four times). Strewn slides were prepared by sampling the suspended residue with a pipette, spreading it on a $22-\times 30-\mathrm{mm}$ cover glass, drying on a hot plate, and mounting in Hyrax.
In selected intervals, one or two samples per 1.5-m section were examined. At least one entire slide was scanned under the light microscope at $500 \times$, and identification of species was checked at $1250 \times$.

The preservation of the diatom assemblages $(\operatorname{good}=\mathrm{G}$; $\bmod$ erate $=\mathrm{M}$; poor $=\mathrm{P}$ ) was estimated based on the degree of breakage and dissolution of the valves and the relative abundance of dissolution-resistant species vs. finely silicified species.

Relative abundance of the various taxa represented on the occurrence charts is reported as abundant (A) if at least 20 specimens are present in one horizontal traverse at $500 \times$, common (C) if 19 to 3 specimens are present in each traverse, few (F) if 1 or 2 specimens are present in each traverse, and rare (R) if fewer than 1 specimen is encountered in each traverse.

\section{RESULTS AND DISCUSSION \\ Late Pliocene-Quaternary}

\section{Sediments}

The upper Neogene sediments at Site 758 are primarily pelagic calcium carbonate, but terrigenous clays and volcanic tephra are important components. Poor to well-preserved diatom assem- 
blages occur in the upper $35 \mathrm{~m}$ of the section at this site (Cores 121-758A-1H to 121-758A-5H; Holocene to upper Pliocene).

The sediments have been double cored (APC) in the upper Neogene of Site 758 (Holes 758A and 758B). Although the APC recovery was excellent $(>100 \%)$, interhole correlation shows sediment recovery gaps ( $1 \mathrm{~m}$ in average) between cores (Farrell and Janecek, this volume). A composite section for Site 758 was constructed based on a combination of Holes 758A and 758B (Farrell and Janecek, this volume), which resulted in a complete sedimentary record for the last $7 \mathrm{~m}$.y. This composite section and composite depths should be used for any high-resolution study. However, because a high-resolution diatom study was not intended in this chapter, I used the depths presented in Peirce, Weissel, et al. (1989) rather than using composite depths (Farrell and Janecek, this volume) for the studied samples and the magnetic reversals. For the same reason, the present study is limited to Hole $758 \mathrm{~A}$.

\section{Diatom Occurrences}

The composition of the diatom assemblages is plotted on Table 1 and vertical ranges of selected taxa are illustrated on Figure 2. Illustration of most of the species recorded in the late Neogene of Hole 758A can be found in Schrader (1974) and Barron (1980) and no additional illustration is provided in this chapter.

\section{Diatom Zonation}

Burckle (1972) proposed a late Neogene to Quaternary diatom zonation for the equatorial Pacific with direct ties to magnetic stratigraphy (Fig. 3). Schrader (1974) and Kazarina (1975) developed late Neogene to Quaternary diatom zonations for the tropical Indian Ocean. Kazarina's zonation is very similar to Burckle's. Schrader's zonation also has close similarities to that of Burckle, especially for the late Pliocene and Quaternary. However, age discrepancies in Schrader's zonation in making core-to-core correlations have been pointed out by Burckle and Opdyke (1977), and Burckle and Opdyke (1977) demonstrated the applicability of Burckle's (1972) equatorial Pacific zonation to the tropical Indian Ocean. Burckle's (1972) zonation has proven to be useful not only in the equatorial Pacific but also in low-latitude regions of other oceans and is used in the low latitudes by most diatom workers (Barron, 1985c).

At Site 758 the composition of the diatom assemblages, as well as the age of the diatom datum levels, are similar enough with respect to the late Neogene and Quaternary in the equatorial Pacific diatom sequence that Burckle's (1972) zonation for the equatorial Pacific can be applied (Fig. 3).

\section{Datum Levels}

Upper Neogene and Quaternary sediments of Site 758 preserved an excellent magnetostratigraphy (Pierce, Weissel, et al., 1989; Farrell and Janecek, this volume). The age of several diatom datum levels (Table 2) is calculated by interpolation from the sedimentation rate curve obtained from the depth (Peirce, Weissel, et al., 1989) and age (Berggren et al., 1985) of the paleomagnetic reversals in Hole 758A, and is compared with datum levels from the equatorial Pacific (Barron, 1985b, in press) and the North Atlantic (Baldauf, 1987). Some of these datum levels are discussed in the subsequent stratigraphy section.

\section{Diatom Biostratigraphy}

\section{Pseudoeunotia doliolus Zone}

The Pseudoeunotia doliolus Zone of Burckle (1972) is recognized from the top of Hole 758 A to Sample 121-758A-2H-6, $67-69 \mathrm{~cm}$, just above the last occurrence of Nitzschia reinholdii (Sample 121-758A-2H-7, 65-67 cm).
The last occurrence of Nitzschia reinholdii correlates in Hole $758 \mathrm{~A}$ with the middle portion of the Matuyama Chronozone and has an estimated age of 0.9 to $1.0 \mathrm{Ma}$ (Table 2). In the equatorial Pacific and North Atlantic oceans this event is calibrated to the lower Brunhes Chronozone and has estimated ages of 0.65 and $0.44 \mathrm{Ma}$, respectively (Barron, 1985b; Baldauf, 1987). As a consequence, the base of the Pseudoeunotia doliolus Zone is significantly older at Site 758 than in the equatorial Pacific and North Atlantic oceans.

Several stratigraphically useful events occur in the Pseudoeunotia doliolus Zone (Table 2):

1. Nitzschia fossilis has its last occurrence in the lower Brunhes Chronozone at an estimated age of $0.44-0.58 \mathrm{Ma}$. This event has a similar age in the North Atlantic $(0.58-0.60 \mathrm{Ma}$; Baldauf, 1987) and in the North Pacific (0.6 Ma; Koizumi and Yanagisawa, 1990). However, it is slightly older in the equatorial Pacific $(0.85$ $\mathrm{Ma}$; Barron, 1985b, in press). The last common occurrence of Nitzschia fossilis at Site 758 between 0.83 and $0.90 \mathrm{Ma}$ is contemporaneous with the last occurrence of this species in the equatorial Pacific.

2. Mesocena quadrangula is a silicoflagellate whose last occurrence is situated between the top of the Jaramillo magnetic polarity event and the base of the Brunhes Chronozone in the equatorial Pacific (Saito and Burckle, 1977; Burckle, 1977) and around $0.73-0.75 \mathrm{Ma}$ in the North Atlantic (Baldauf, 1987). The last occurrence of Mesocena quadrangula in Hole 758 A has a similar stratigraphic occurrence and has an estimated age of $0.75-0.83 \mathrm{Ma}$.

\section{Nitzschia reinholdii Zone}

The Nitzschia reinholdii Zone of Burckle (1972) occurs from Sample 121-758A-2H-7, 65-67 cm (last occurrence of Nitzschia reinholdii), to Sample 121-758A-4H-3, 65-67 cm (first occurrence of Pseudoeunotia doliolus). This zone can be subdivided in two subzones based on the last occurrence of Rhizosolenia praebergonii in Sample 121-758A-3H-6, 65-67 cm (Subzone B, Samples $121-758 \mathrm{~A}-2 \mathrm{H}-7,65-67 \mathrm{~cm}$, to $121-758 \mathrm{~A}-3 \mathrm{H}-5,65-67 \mathrm{~cm}$; Subzone A, Samples 121-758A-3H-6, $65-67 \mathrm{~cm}$, to $121-758 \mathrm{~A}$ $4 \mathrm{H}-3,65-67 \mathrm{~cm})$.

Several stratigraphically useful events occur in the Nitzschia reinholdii Zone (Table 2 ):

1. The last occurrence of Nitzschia reinholdii at Site 758 falls between 0.9 and 1.0 Ma. In the equatorial Pacific and in the North Atlantic, this datum level is significantly younger (cf. discussion above).

2. Rhizosolenia praebergonii var. robusta and Rhizosolenia praebergonii var. praebergonii have their last occurrence at Site 758 in the middle portion of the Matuyama Chronozone at an estimated age of 1.49-1.60 Ma. This is contemporaneous with the last occurrence of $R$. praebergonii var. robusta in the equatorial Pacific (1.55 Ma; Barron, 1985b, in press). However, R. praebergonii var. praebergonii has it last occurrence at $1.85 \mathrm{Ma}$ in the equatorial Pacific (Barron, 1985b, in press); thus, this variety seems to survive longer in the tropical Indian Ocean.

3. Pseudoeunotia doliolus has its first occurrence at Site 758 in the lower portion of the Matuyama Chronozone at an estimated age of 1.85-2.20 Ma. It appears to be slightly older than its age in the equatorial Pacific (1.80 Ma; Barron, 1985b, in press) and in the North Atlantic (1.84 Ma; Baldauf, 1987), and possibly similar to its first occurrence in the western subtropical North Pacific (2.0 Ma; Koizumi and Yanagisawa, 1990).

\section{Rhizosolenia praebergonii Zone}

The Rhizosolenia praebergonii Zone of Burckle (1972) is recognized from Sample 121-758A-4H-4, 66-68 cm (sample immediately below the sample containing the first occurrence of 


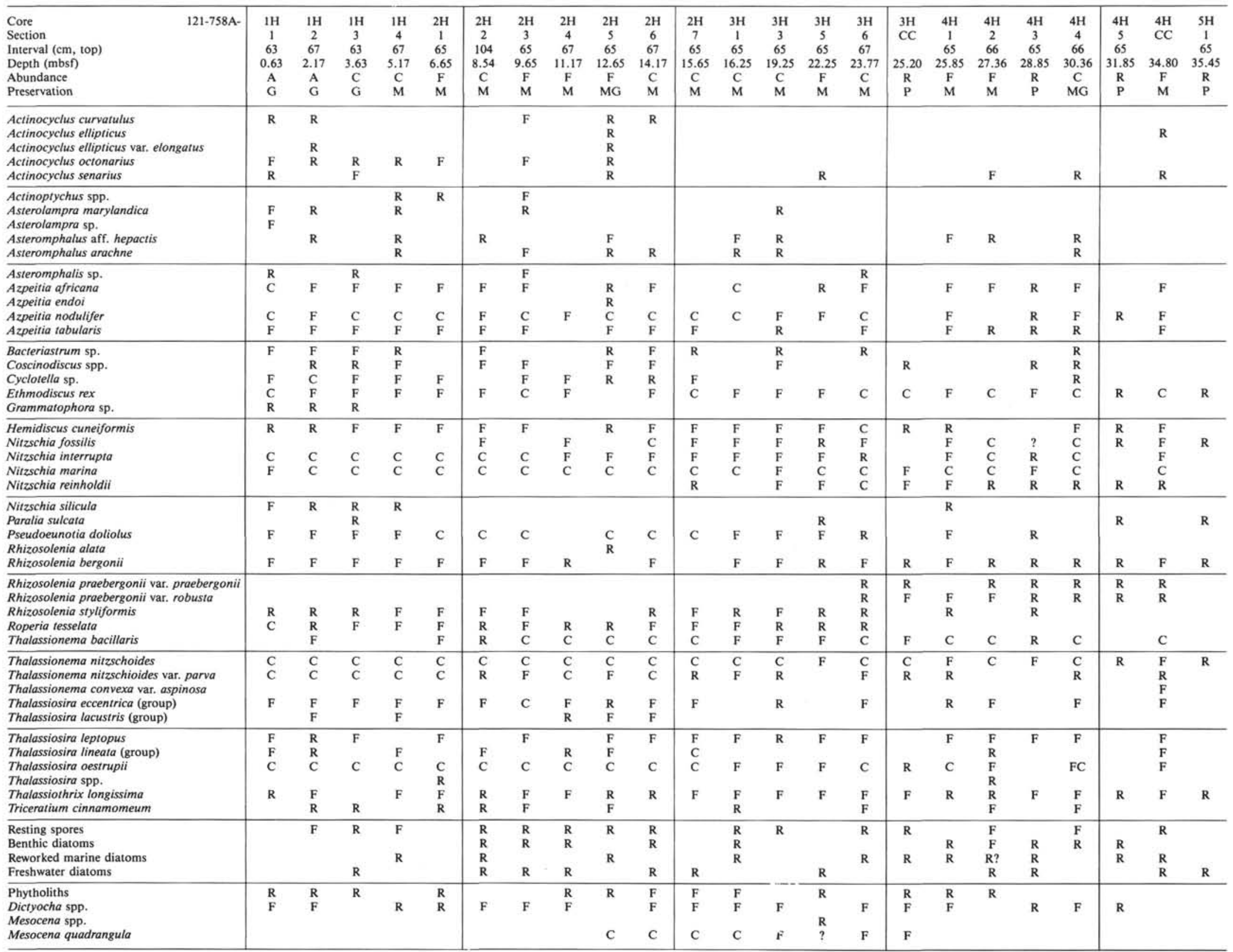

Note: $\mathrm{P}=$ poor $; \mathrm{M}=$ moderate $\mathrm{G}=$ good $; \mathrm{A}=$ abundant $\mathrm{C}=$ common; $\mathrm{F}=$ few; $\mathrm{R}=$ rare. 


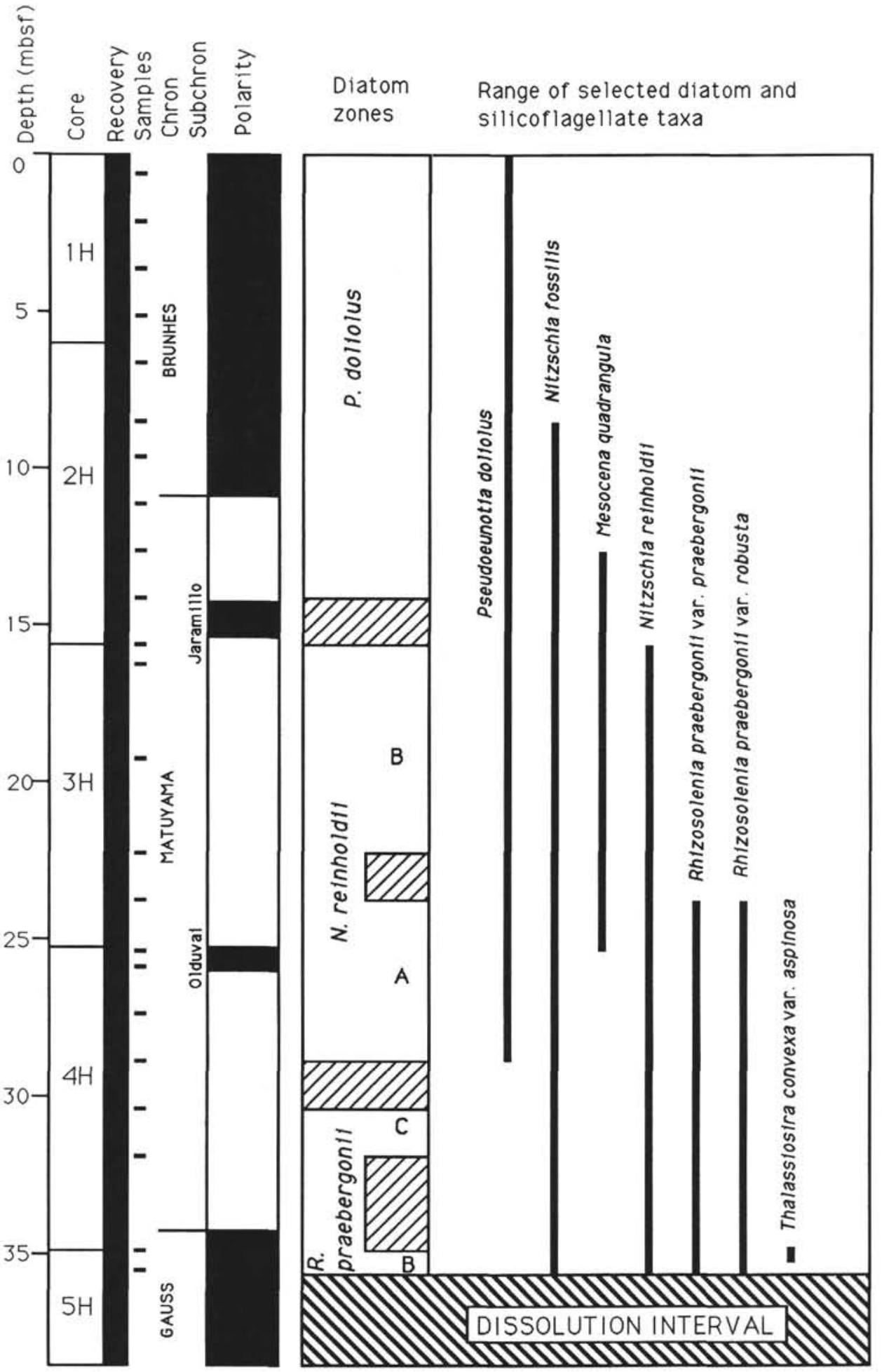

Figure 2. Diatom stratigraphy and ranges of selected diatom and silicoflagellate taxa from the top of Hole 758A down to Core 121-758A-5H (Holocene to late Pliocene). Magnetostratigraphy according to Peirce, Weissel, et al. (1989). Diagonal lines represent intervals of uncertainty (inclined to right, interval between samples; inclined to left, dissolution). 


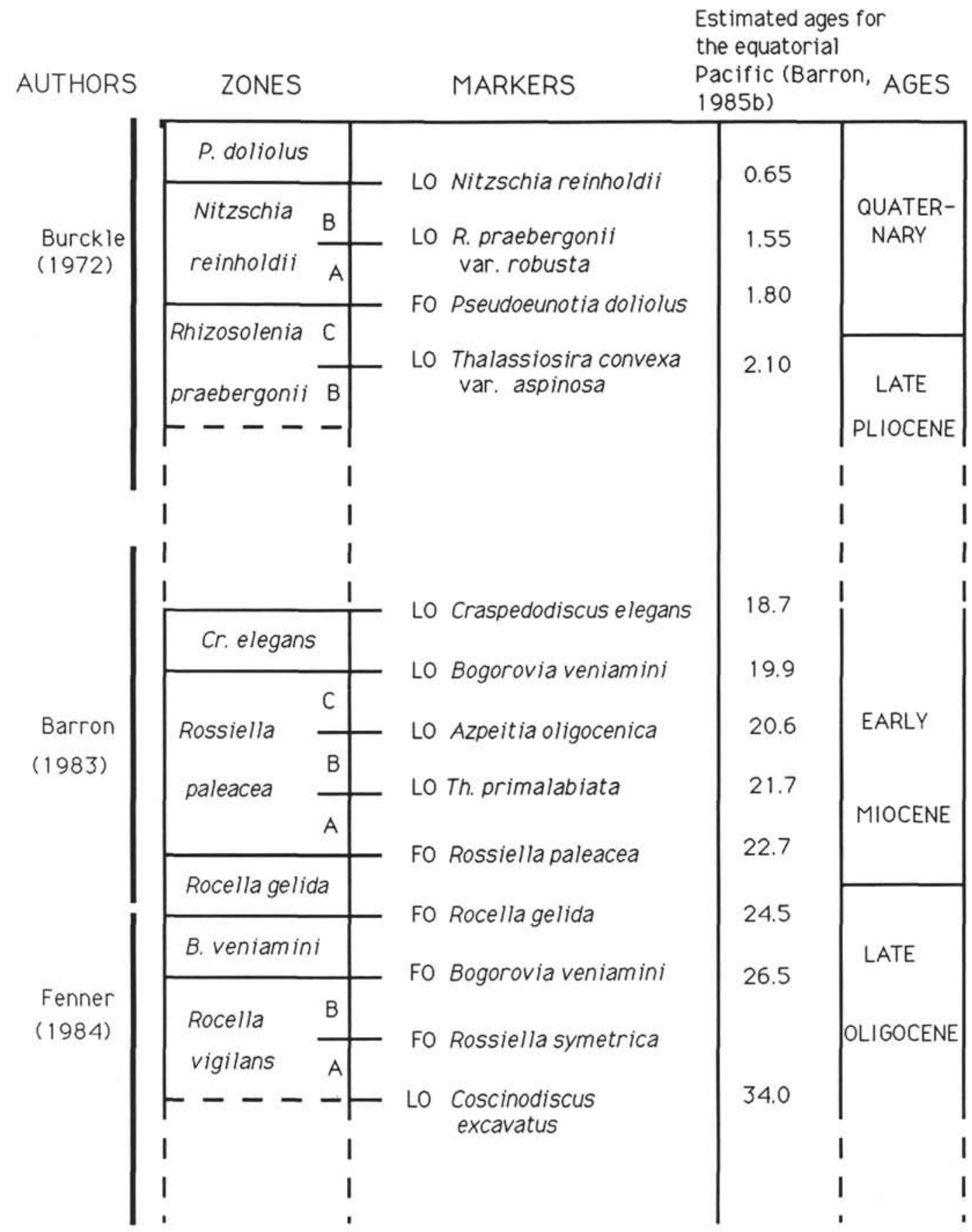

Figure 3. Diatom zonation used in this chapter. Burckle's (1972) zonation is used for the upper Pliocene-Quaternary interval, 'Barron's (1983) zonation is used for the lower Miocene, and Fenner's (1984) zonation is used for the upper Oligocene. Ages of the zonal boundaries for the equatorial Pacific Ocean after Barron (1985b). $\mathrm{FO}=$ first occurrence; $\mathrm{LO}=$ last occurrence.

Pseudoeunotia doliolus) to Sample 121-758A-4H-CC. From Core 121-758 A-5H down to Core 121-758A-16X diatoms are sparse to absent and no zonation was developed for this interval.

The Subzone $\mathrm{C}$ of Rhizosolenia praebergonii Zone is recognized between Sample 121-758A-4H-3, 65-67 cm, to Sample 121-758A-4H-5, 65-67 cm (sample immediately above the last occurrence of Thalassiosira convexa var. aspinosa at Sample 121-758A-4H-CC). Sample 121-758A-4H-CC is therefore as- signed to Subzone B of the Rhizosolenia praebergonii Zone. Due to preservation loss, diatom zonation is not attempted below that sample.

The last occurrence of Thalassiosira convexa var. aspinosa lies at Site 758 in the upper Gauss Chronozone at an estimated age of 2.20-2.51 Ma. This is similar to the last occurrence of this species in the equatorial Pacific (2.10 Ma; Barron 1985b, in press) and in the North Atlantic (2.3-2.5 Ma; Baldauf, 1987). 
Table 2. Depth and age of several diatom datums for the upper Pliocene to Quaternary interval in Hole 758A.

\begin{tabular}{|c|c|c|c|c|c|}
\hline \multirow[b]{2}{*}{ Diatom datums } & \multirow[b]{2}{*}{ Interval } & \multirow[b]{2}{*}{$\begin{array}{c}\text { Depth range } \\
\text { (mbsf) }\end{array}$} & \multirow[b]{2}{*}{$\begin{array}{l}\text { Age } \\
\text { (Ma) }\end{array}$} & \multirow{2}{*}{$\begin{array}{c}\text { Equatorial } \\
\text { Pacific } \\
\text { Barron, } \\
\text { in press } \\
\text { Age (Ma) }\end{array}$} & \multirow{2}{*}{$\begin{array}{c}\text { North } \\
\text { Atlantic } \\
\text { Baldauf, } \\
1987 \\
\text { Age (Ma) }\end{array}$} \\
\hline & & & & & \\
\hline & $1-758 \mathrm{~A}-$ & & & & \\
\hline LO Nitzschia fossilis & $2 \mathrm{H}-1,65-67 \mathrm{~cm}$, to $2 \mathrm{H}-2,104-106 \mathrm{~cm}$ & $6.65-8.54$ & $0.44-0.58$ & 0.85 & $0.58-0.60$ \\
\hline LCO Nitzschia fossilis & $2 \mathrm{H}-5,65-67 \mathrm{~cm}$, to $2 \mathrm{H}-6,67-69 \mathrm{~cm}$ & $12.65-14.17$ & $0.83-0.90$ & & \\
\hline LO Mesocena quadrangula & $2 \mathrm{H}-4,67-69 \mathrm{~cm}$, to $2 \mathrm{H}-5,65-67 \mathrm{~cm}$ & $11.17-12.65$ & $0.75-0.83$ & 0.79 & $0.73-0.75$ \\
\hline LO Nitzschia reinholdii & $2 \mathrm{H}-6,67-69 \mathrm{~cm}$, to $2 \mathrm{H}-7,65-67 \mathrm{~cm}$ & $14.17-15.65$ & $0.90-1.0$ & 0.65 & 0.44 \\
\hline LCO Nitzschia reinholdii & $2 \mathrm{H}-7,65-67 \mathrm{~cm}$, to $3 \mathrm{H}-3,65-67 \mathrm{~cm}$ & $15.65-19.25$ & $1.00-1.28$ & & \\
\hline $\begin{array}{l}\text { LO Rhizosolenia praebergonii } \\
\text { v. robusta }\end{array}$ & $3 \mathrm{H}-5,65-67 \mathrm{~cm}$, to $3 \mathrm{H}-6,67-69 \mathrm{~cm}$ & $22.25-23.77$ & $1.49-1.60$ & 1.55 & \\
\hline LO Rhizosolenia praebergonii & $3 \mathrm{H}-5,65-67 \mathrm{~cm}$, to $3 \mathrm{H}-6,67-69 \mathrm{~cm}$ & $22.25-23.77$ & $1.49-1.60$ & 1.85 & \\
\hline FO Pseudoeunotia doliolus & $4 \mathrm{H}-3,65-67 \mathrm{~cm}$, to $4 \mathrm{H}-4,66-68 \mathrm{~cm}$ & $25.85-30.36$ & $1.85-2.20$ & 1.80 & 1.84 \\
\hline LO Thalassiosira convexa & $4 \mathrm{H}-4,66-68 \mathrm{~cm}$, to $4 \mathrm{H}-\mathrm{CC}$ & $30.36-34.80$ & $2.20-2.51$ & 2.10 & $2.3-2.5$ \\
\hline
\end{tabular}

Note: Comparison with similar datums in the equatorial Pacific (Barron, 1985b, in press) and in the North Atlantic (Baldauf, 1987). $\mathrm{LO}=$ last occurrence; $\mathrm{LCO}=$ last common occurrence; $\mathrm{FO}=$ first occurrence.

\section{Conclusion}

This study confirms the possibility of using (in the low-latitude Indian Ocean) the low-latitude diatom zonation of Burckle (1972), which was established in the equatorial Pacific. The synchroneity between the equatorial Pacific, the North Atlantic and the equatorial Indian Ocean of several Plio-Quaternary diatom datum levels is tested, and good synchroneity for 4 out of the 7 considered events is shown.

\section{Late Oligocene-Early Miocene}

Diatomaceous sediments of late Oligocene and early Miocene age were recovered at Site 758 between Cores 121-758A-16X and $-24 X$. Limiting factors encountered in this study are: (1) the absence of magnetic stratigraphy in this interval, (2) the presence of a hiatus at the Miocene/Oligocene boundary, and (3) the poor preservation of the diatoms in this interval. However, the assemblages are documented and a biostratigraphy is established for this diatomaceous interval.

Table 3 lists the diatom content of the 34 samples studied between Cores 121-758A-16X and $-24 \mathrm{X}$. Figure 4 reports the ranges of selected diatom taxa. Numerous diatom taxa are illustrated Plates 1-4.

\section{Diatom Zonation}

There are enough similarities between the diatom flora from Site 758 and those described from other low-latitude sections to enable the use of the previously established zonations of Barron (1983) for the early Miocene and Fenner (1984) for the late Oligocene (see Fig. 3).

\section{Diatom Biostratigraphy}

Craspedodiscus elegans Zone (18.7 to $19.9 \mathrm{Ma}$ )

The Craspedodiscus elegans Zone of Barron (1983) is tentatively recognized between Sample 121-758A-16X-CC and Sample 121-758A-17X-3, 65-67 cm, based on the last occurrence of Bogorovia veniamini in Sample 121-758A-17X-CC. The top of the zone is not formally recognized in Hole 758A, as Craspedodiscus elegans is very sporadic in the sequence. However, the occurrence of Thalassiosira fraga and the absence of Triceratium pileus in Sample 121-758A-16X-CC suggests that it belongs to the Craspedodiscus elegans Zone. In the equatorial Pacific Nitzschia maleinterpretaria first occurs at 18.8 Ma near the top of the Craspedodiscus elegans Zone (Barron, 1985b). The occurrence of Nitzschia aff. maleinterpretaria in Sample 121-758A-
$16 \mathrm{X}-\mathrm{CC}$ suggests that it might approximate the top of the Craspedodiscus elegans Zone at Site 758.

Characteristic Flora. Some characteristic taxa include Thalassiosira fraga (first occurrence in Sample 121-758A-17X-3, 65-67 $\mathrm{cm})$, Thalassiosira spinosa, Thalassiosira spumellaroides, Cestodiscus spp., Actinocyclus aff. ellipticus, and Coscinodiscus sawamurae (first occurrence in Sample 121-758A-17X-2, 65-67 cm).

Rossiella paleacea Zone (19.9 to $22.7 \mathrm{Ma}$ )

The Rossiella paleacea Zone of Barron (1983) is recognized between Sample 121-758A-17X-CC (last occurrence of Bogorovia veniamini) and Sample 121-758A-21X-3, 102-104 cm (first occurrence of Rossiella paleacea). As defined in the equatorial Pacific (Barron, 1983), the Rossiella paleacea Zone is divided in 3 subzones using the last occurrence of Azpeitia oligocenica (boundary $\mathrm{C} / \mathrm{B}$ ) and the last occurrence of Thalassiosira primalabiata (boundary B/A). These subzones are not formally recognized in Hole $758 \mathrm{~A}$ as Azpeitia oligocenica is very sporadic in the Miocene of the section and only one questionable specimen of Thalassiosira primalabiata has been found in Sample 121$758 \mathrm{~A}-21 \mathrm{X}-3,102-104 \mathrm{~cm}$. Some tentative indication of the stratigraphic position of the different subzones at Site 758 may be suggested using the ranges of equatorial Pacific secondary stratigraphic markers (Barron, 1983, 1985b). As such, the last consistent occurrence of Melosira architecturalis in Sample 121-758A-19-CC suggests the correlation of that sample with the Subzone B. In addition, Barron (1983) reported the last occurrence of Coscinodiscus lewisianus var. rhomboides (as Coscinodiscus lewisianus var. similis) at the base of the Subzone A at DSDP Site 71. Therefore, the last occurrence of Coscinodiscus lewisianus var. rhomboides in Sample 121-758A-21X-1, 65-67 $\mathrm{cm}$ would place that sample in the lower part of Subzone A. In conclusion, it is suggested that the Rossiella paleacea Zone may be rather complete at Site 758 .

Characteristic Flora. Some characteristic taxa in this zone include Asteromphalus oligocenicus, Bogorovia veniamini, Cestodiscus pulchellus, Coscinodiscus rhombicus, Synedra jouseana, Rossiella paleacea, and Rossiella symmetrica.

Remarks on the Stratigraphic Position of Section 121-758A$21 X-4$. Samples in this section are located beneath the first occurrence of Rossiella paleacea. However, they do not contain Rocella gelida, a cosmopolitan, highly silicified species whose last occurrence coincides with the first occurrence of Rossiella 


\section{E. FOURTANIER}

Table 3. Occurrence of diatom species and Macrora stella (incertae sedis) in Hole 758A for the upper Oligocene and lower Miocene interval.

\begin{tabular}{|c|c|c|c|c|c|c|c|c|c|c|c|c|c|c|}
\hline $\begin{array}{l}\text { Core } \\
\text { Section } \\
\text { Interval (cm, top) } \\
\text { Depth (mbsf) } \\
\text { Abundance } \\
\text { Preservation }\end{array}$ & $\begin{array}{c}16 \mathrm{X} \\
\mathrm{CC} \\
146.99 \\
\mathrm{R} \\
\mathrm{P}\end{array}$ & $\begin{array}{c}17 \mathrm{X} \\
1 \\
65 \\
151.35 \\
\mathrm{~F} \\
\mathrm{M}\end{array}$ & $\begin{array}{c}17 \mathrm{X} \\
2 \\
65 \\
152.85 \\
\mathrm{~F} \\
\mathrm{P}\end{array}$ & $\begin{array}{c}17 \mathrm{X} \\
3 \\
65 \\
154.35 \\
\mathrm{~F} \\
\mathrm{M}\end{array}$ & $\begin{array}{l}17 X \\
C C \\
156.35 \\
F \\
M\end{array}$ & $\begin{array}{c}18 \mathrm{X} \\
1 \\
65 \\
161.05 \\
\mathrm{~F} \\
\mathrm{M}\end{array}$ & $\begin{array}{c}18 \mathrm{X} \\
3 \\
65 \\
164.05 \\
\mathrm{C} \\
\mathrm{G}\end{array}$ & $\begin{array}{c}18 \mathrm{X} \\
4 \\
65 \\
165.55 \\
\mathrm{~A} \\
\mathrm{GM}\end{array}$ & $\begin{array}{c}18 X \\
\text { CC } \\
166.57 \\
\text { C } \\
\text { G }\end{array}$ & $\begin{array}{c}19 X \\
1 \\
65 \\
170.65 \\
\text { C } \\
M\end{array}$ & $\begin{array}{c}19 \mathrm{X} \\
3 \\
65 \\
173.65 \\
\mathrm{~F} \\
\mathrm{M}\end{array}$ & $\begin{array}{c}19 X \\
4 \\
65 \\
175.15 \\
C \\
\text { MG }\end{array}$ & $\begin{array}{c}19 \mathrm{X} \\
\mathrm{CC} \\
176.83 \\
\mathrm{C} \\
\mathrm{G}\end{array}$ & $\begin{array}{c}20 \mathrm{X} \\
1 \\
65 \\
180.35 \\
\mathrm{R} \\
\mathrm{P}\end{array}$ \\
\hline $\begin{array}{l}\text { Actinocyclus aff. ellipticus } \\
\text { Actinocyclus octonarius } \\
\text { Actinoptychus senarius } \\
\text { Asterolampra aff. acutiloba } \\
\text { Asterolampra insignis grp. }\end{array}$ & & $\mathbf{R}$ & $\mathbf{R}$ & $\mathrm{R}$ ? & R & $\mathbf{R}$ & $R$ & $\begin{array}{l}\mathrm{R} \\
\mathrm{R}\end{array}$ & & & R & $\begin{array}{l}R \\
R\end{array}$ & & $?$ \\
\hline $\begin{array}{l}\text { Asterolampra marylandica } \\
\text { Asterolampra sp. } \\
\text { Asteromphalus oligocenicus } \\
\text { Asteromphalus sp. } \\
\text { Azpeitia aff. T. bukryi } \\
\end{array}$ & & $\begin{array}{l}\mathrm{R} \\
\mathrm{R}\end{array}$ & $\mathrm{R}$ ? & $\mathbf{R}$ & $\mathrm{F}$ & $\begin{array}{l}\mathrm{R} \\
\mathrm{R}\end{array}$ & $\begin{array}{l}F \\
R\end{array}$ & $\begin{array}{c}\mathrm{F} \\
\mathrm{R} ?\end{array}$ & $\begin{array}{l}\mathrm{R} \\
\mathrm{R} \\
\mathrm{R}\end{array}$ & $\begin{array}{l}\mathrm{F} \\
\mathrm{R}\end{array}$ & R & $\begin{array}{l}\mathrm{R} \\
\mathrm{R}\end{array}$ & $\mathrm{F}$ & $\begin{array}{l}\mathrm{F} \\
\mathrm{R}\end{array}$ \\
\hline $\begin{array}{l}\text { Azpeitia nodulifer var. } \\
\text { Azpeitia oligocenica } \\
\text { Azpeitia praenodulifera } \\
\text { Azpeitia salisburyana } \\
\text { Azpeitia spp. } \\
\end{array}$ & & $\begin{array}{l}\mathrm{R} \\
\mathrm{F}\end{array}$ & F & & $\begin{array}{l}\mathbf{R} \\
\mathbf{R} \\
\mathrm{R}\end{array}$ & $\begin{array}{l}\mathrm{R} \\
\mathrm{R} \\
\mathrm{F}\end{array}$ & $\begin{array}{l}\mathrm{R} \\
\mathrm{F}\end{array}$ & $\begin{array}{l}\mathrm{F} \\
?\end{array}$ & $\begin{array}{l}\mathrm{R} ? \\
\mathrm{~F}\end{array}$ & $\mathbf{R}$ & $\mathbf{R}$ & R & $F$ & \\
\hline $\begin{array}{l}\text { Azpeitia tabularis } \\
\text { Bacteriastrum sp. } \\
\text { Baxteriopsis brunii } \\
\text { Bog. intermed. veniamini/symmetrica } \\
\text { Bogorovia sp. } 1 \text { (small forms) } \\
\end{array}$ & $R$ & $\mathrm{~F}$ & $\mathbf{R}$ & $\mathbf{R}$ & & $\mathbf{R}$ & & $\mathbf{R}$ & $R$ & $?$ & $?$ & $\begin{array}{c}\mathrm{F} \\
\mathrm{R} \\
\mathrm{R} ?\end{array}$ & $\mathbf{R}$ & R \\
\hline $\begin{array}{l}\text { Bogorovia veniamini } \\
\text { Cestodiscus japonicus? } \\
\text { Cestodiscus kugleri } \\
\text { Cestodiscus ovalis } \\
\text { Cestodiscus spp. }\end{array}$ & $R$ ? & $\begin{array}{l}R \\
F\end{array}$ & $\mathrm{~F}$ & $\begin{array}{l}\mathrm{R} \\
\mathrm{F}\end{array}$ & $\begin{array}{l}\mathrm{R} \\
\mathrm{R}\end{array}$ & $\begin{array}{l}\mathrm{R} ? \\
\mathrm{~F} \\
\mathrm{~F} \\
\mathrm{~F}\end{array}$ & $\begin{array}{l}\mathrm{F} \\
\mathrm{F} \\
\mathrm{F}\end{array}$ & $\begin{array}{l}\mathrm{F} \\
\mathrm{F} \\
\mathrm{F}\end{array}$ & $\begin{array}{l}\mathrm{F} \\
\mathrm{R} \\
\mathrm{F} \\
\mathrm{F}\end{array}$ & $\begin{array}{l}\mathrm{F} \\
\mathrm{R} \\
\mathrm{F}\end{array}$ & F & $\begin{array}{l}\mathrm{F} \\
\mathrm{R} \\
\mathrm{F}\end{array}$ & $\begin{array}{l}\mathrm{R} \\
\mathrm{F} \\
\mathrm{R}\end{array}$ & R \\
\hline $\begin{array}{l}\text { Cestodiscus sp. } 1 \\
\text { Cestodiscus sp. } 3 \\
\text { Cestodiscus sp. } 4 \\
\text { Coscinodiscus lewisiana } \\
\text { Coscinodiscus lewisianus v. rhomboides }\end{array}$ & & $\mathbf{R}$ & R & $\mathbf{R}$ & $\begin{array}{l}\mathrm{R} \\
\mathrm{R}\end{array}$ & $\begin{array}{c}\mathrm{RF} \\
\mathrm{R} \\
\mathrm{R}\end{array}$ & $\begin{array}{l}\mathrm{R} \\
\mathrm{F}\end{array}$ & & R & & & & & \\
\hline $\begin{array}{l}\text { Coscinodiscus marginatus } \\
\text { Coscinodiscus rhombicus } \\
\text { Coscinodiscus sawamurae } \\
\text { Coscinodiscus spp. } \\
\text { Craspedodiscus barronii }\end{array}$ & $\begin{array}{l}F \\
R\end{array}$ & $\begin{array}{l}\mathrm{R} \\
\mathrm{F} \\
\mathrm{R} \\
\mathrm{F} \\
\mathrm{R}\end{array}$ & $\begin{array}{l}\mathrm{R} \\
\mathrm{F} \\
\mathrm{R}\end{array}$ & $\begin{array}{l}R \\
F\end{array}$ & $\begin{array}{l}\mathrm{F} \\
\mathrm{R}\end{array}$ & $\begin{array}{l}\mathrm{R} \\
\mathrm{F} \\
\mathrm{R}\end{array}$ & $\begin{array}{l}\mathrm{R} \\
\mathrm{F} \\
\mathrm{R}\end{array}$ & $\begin{array}{l}\mathrm{R} \\
\mathrm{C}\end{array}$ & $\begin{array}{l}\mathrm{R} \\
\mathrm{C} \\
\mathrm{R}\end{array}$ & $\begin{array}{l}\mathrm{R} \\
\mathrm{F} \\
\mathrm{F} \\
\mathrm{R}\end{array}$ & $\begin{array}{l}R \\
F \\
F\end{array}$ & $\begin{array}{l}\mathrm{R} \\
\mathrm{F} \\
\mathrm{R} \\
\mathrm{F}\end{array}$ & $\begin{array}{l}\mathrm{R} \\
\mathrm{F} \\
\mathrm{F} \\
\mathrm{R}\end{array}$ & $\begin{array}{l}F \\
F\end{array}$ \\
\hline $\begin{array}{l}\text { Craspedodiscus elegans } \\
\text { Craspedodiscus elegans v. assymmetricus } \\
\text { Dimerogamma aff. fulvum } \\
\text { Ethmodiscus sp. } \\
\text { Grammatophora sp. }\end{array}$ & $\mathrm{F}$ & $\begin{array}{c}? \\
\mathrm{R} ? \\
\mathrm{R}\end{array}$ & $\mathrm{F}$ & $\begin{array}{l}\mathrm{R} \\
\mathrm{R}\end{array}$ & F & $\begin{array}{l}\mathrm{R} ? \\
\mathrm{R} \\
\mathrm{R}\end{array}$ & $\begin{array}{l}\mathrm{R} \\
\mathrm{R} \\
\mathrm{R} \\
\mathrm{R}\end{array}$ & $\begin{array}{l}\mathrm{R} \\
\mathrm{F}\end{array}$ & $\begin{array}{l}R \\
R \\
F\end{array}$ & $\begin{array}{l}\mathrm{R} ? \\
\mathrm{R} \\
\mathrm{F}\end{array}$ & F & $F$ & $\begin{array}{l}\mathrm{R} ? \\
\mathrm{~F} \\
\mathrm{R}\end{array}$ & $\mathbf{R}$ \\
\hline $\begin{array}{l}\text { Hemiaulus longicornis } \\
\text { Hemiaulus sp. } \\
\text { Hemiaulus sp. } 1 \\
\text { Hemiaulus subacutus } \\
\text { Lisitzinia ornata } \\
\end{array}$ & R & $\begin{array}{l}\mathrm{F} \\
\mathrm{R}\end{array}$ & $\mathrm{F}$ & $\mathbf{R}$ & F & $\mathrm{F}$ & F & $\begin{array}{l}\mathrm{R} \\
\mathrm{F}\end{array}$ & F & $\mathrm{F}$ & $F$ & $\begin{array}{l}\mathrm{R} \\
\mathrm{F}\end{array}$ & C & F \\
\hline $\begin{array}{l}\text { Melosira architecturalis } \\
\text { Nitzschia aff. maleinterpretaria } \\
\text { Nitzschia pusilla } \\
\text { Paralia sulcata } \\
\text { Rhaphidodiscus marylandicus } \\
\end{array}$ & $\begin{array}{l}\mathrm{R} \\
\mathrm{R} \\
\mathrm{R} \\
\end{array}$ & & $\begin{array}{l}\mathrm{R} ? \\
\mathrm{R}\end{array}$ & $\mathrm{R}$ & $\begin{array}{l}R \\
R\end{array}$ & $\begin{array}{l}R \\
R \\
\end{array}$ & $\mathrm{R}$ & & $\mathbf{R}$ & $\begin{array}{l}\mathrm{R} \\
\mathrm{R}\end{array}$ & & $\mathbf{R}$ & $\begin{array}{l}\mathrm{F} \\
\mathrm{R} ?\end{array}$ & \\
\hline $\begin{array}{l}\text { Rhaphoneis sp. } \\
\text { Rhizosolenia firma } \\
\text { Rhizosolenia sp. aff. alata } \\
\text { Rhizosolenia sp. (hebetata group) } \\
\text { Rocella princeps }\end{array}$ & $\mathbf{R}$ & & & $\mathbf{R}$ & & $\begin{array}{l}\mathrm{R} \\
\mathrm{R}\end{array}$ & & $\mathbf{R}$ & $\begin{array}{l}R \\
R\end{array}$ & & R & $\begin{array}{l}\mathbf{R} \\
\mathrm{R}\end{array}$ & & \\
\hline $\begin{array}{l}\text { Rocella vigilans } \\
\text { Rossiella magna } \\
\text { Rossiella paleacea } \\
\text { Rossiella symmetrica } \\
\text { Rouxia quadrangula } \\
\end{array}$ & $\mathrm{R}$ & $\begin{array}{l}\mathrm{R} ? \\
\mathrm{R}\end{array}$ & & $\begin{array}{l}\mathbf{R} \\
\mathbf{R}\end{array}$ & $\begin{array}{l}\mathrm{F} \\
\mathrm{R} \\
\mathrm{R}\end{array}$ & $\begin{array}{l}\mathrm{F} \\
\mathrm{F} \\
\mathrm{F} \\
\mathrm{R}\end{array}$ & $\begin{array}{l}\mathrm{R} \\
\mathrm{F} \\
\mathrm{R} \\
\mathrm{R}\end{array}$ & $\begin{array}{l}\mathrm{F} \\
\mathrm{F} \\
\mathrm{R}\end{array}$ & $\begin{array}{l}\mathrm{R} \\
\mathrm{F} \\
\mathrm{F} \\
\mathrm{R}\end{array}$ & $\begin{array}{l}\mathrm{R} \\
\mathrm{R} \\
\mathrm{R} \\
\end{array}$ & $\begin{array}{l}\mathrm{F} \\
\mathrm{R} \\
\mathrm{R}\end{array}$ & $\begin{array}{l}\mathrm{C} \\
\mathrm{C} \\
\mathrm{C} \\
\mathrm{R}\end{array}$ & $\begin{array}{l}\mathrm{F} \\
\mathrm{F} \\
\mathrm{F} \\
\mathrm{R}\end{array}$ & $\begin{array}{l}\mathrm{R} \\
\mathrm{F}\end{array}$ \\
\hline $\begin{array}{l}\text { Sceptroneis sp. } \\
\text { Stellarima sp. } \\
\text { Stephanopyxis sp. } \\
\text { Synedra jouseana } \\
\text { Thalassionema spp. }\end{array}$ & $\begin{array}{l}\mathrm{F} \\
\mathrm{F} \\
\mathrm{R}\end{array}$ & $\begin{array}{l}\mathrm{C} \\
\mathrm{C}\end{array}$ & $\begin{array}{l}F \\
F\end{array}$ & $\begin{array}{l}\mathrm{C} \\
\mathrm{F}\end{array}$ & $\begin{array}{l}\mathrm{R} \\
\mathrm{F} \\
\mathrm{C}\end{array}$ & $\begin{array}{l}\mathrm{C} \\
\mathrm{F}\end{array}$ & $\stackrel{\mathrm{F}}{\mathrm{C}}$ & $\begin{array}{c}\mathrm{AC} \\
\mathrm{C}\end{array}$ & $\begin{array}{l}\mathrm{R} \\
\mathrm{F} \\
\mathrm{C}\end{array}$ & $\begin{array}{l}F \\
F\end{array}$ & $\begin{array}{l}\mathrm{C} \\
\mathrm{F}\end{array}$ & $\begin{array}{l}\mathrm{R} \\
\mathrm{C} \\
\mathrm{C}\end{array}$ & $\begin{array}{l}R \\
R \\
R \\
F \\
F\end{array}$ & $\begin{array}{l}\mathrm{F} \\
\mathrm{F}\end{array}$ \\
\hline $\begin{array}{l}\text { Thalassiosira aff. fraga (fine) } \\
\text { Thalassiosira aff. irregulata } \\
\text { Thalassiosira fraga } \\
\text { Thalassiosira leptopus } \\
\text { Thalassiosira mediaconvexa }\end{array}$ & $\begin{array}{l}R \\
R\end{array}$ & $\begin{array}{l}\mathrm{F} \\
\mathrm{R} \\
\mathrm{R}\end{array}$ & F & $\begin{array}{l}\mathrm{F} \\
\mathrm{R}\end{array}$ & & & & $\mathrm{F}$ & R & & & R & F & \\
\hline $\begin{array}{l}\text { Thalassiosira primalabiata } \\
\text { Thalassiosira spinosa } \\
\text { Thalassiosira spumellaroides } \\
\text { Thalassiosira sp. } \\
\text { Thalassiosira spp. }\end{array}$ & $\mathrm{F}$ & $\begin{array}{l}\mathrm{R} \\
\mathrm{R} \\
\mathrm{C}\end{array}$ & R & $\mathrm{F}$ & $\begin{array}{c}\mathrm{R} \\
\mathrm{R} ? \\
\mathrm{~F}\end{array}$ & $\begin{array}{l}\mathrm{R} \\
\mathbf{R} \\
\mathrm{F}\end{array}$ & $\begin{array}{l}\mathrm{R} ? \\
\mathrm{~F}\end{array}$ & $\begin{array}{l}\mathrm{R} \\
\mathrm{F}\end{array}$ & $\begin{array}{l}\mathrm{R} ? \\
\mathrm{R} \\
\mathrm{F}\end{array}$ & $\begin{array}{l}\mathrm{R} \\
\mathrm{F} \\
\mathrm{F}\end{array}$ & $\mathrm{F}$ & $\begin{array}{l}\mathbf{R} \\
\mathbf{F} \\
\mathrm{F} \\
\mathrm{F}\end{array}$ & $\begin{array}{l}\mathrm{R} \\
\mathrm{F} \\
\mathrm{R} \\
\end{array}$ & $\mathrm{R}$ \\
\hline $\begin{array}{l}\text { Diatom resting spores } \\
\text { Melosira granulata s.I. (freshwater) } \\
\text { Macrora stella (Silicoflagellate) }\end{array}$ & $R$ & $\begin{array}{l}\mathrm{R} \\
\mathrm{R}\end{array}$ & & $\begin{array}{l}\mathbf{R} \\
\mathbf{R}\end{array}$ & $\begin{array}{l}\mathbf{R} \\
\mathbf{R} \\
\mathbf{R}\end{array}$ & $\begin{array}{l}\mathrm{R} \\
\mathrm{F}\end{array}$ & $\begin{array}{l}\mathbf{R} \\
\mathbf{R} \\
\mathbf{R}\end{array}$ & $\begin{array}{l}\mathrm{F} \\
\mathrm{R}\end{array}$ & $\begin{array}{l}\mathrm{R} \\
\mathrm{R} \\
\mathrm{R}\end{array}$ & $\begin{array}{l}\mathrm{F} \\
\mathrm{R} \\
\mathrm{R}\end{array}$ & $\begin{array}{l}\mathrm{R} \\
\mathrm{R} \\
\mathrm{R}\end{array}$ & $\begin{array}{l}\mathrm{F} \\
\mathrm{F} \\
\mathrm{R}\end{array}$ & $\begin{array}{l}R \\
F\end{array}$ & $\mathbf{R}$ \\
\hline
\end{tabular}

Note: $\mathrm{P}=$ poor $\mathrm{M}=$ moderate $\mathrm{G}=$ good $\mathrm{A}=$ abundant $\mathrm{C}=$ common; $\mathrm{F}=$ few; $\mathrm{R}=$ rare. 
Table 3 (continued).

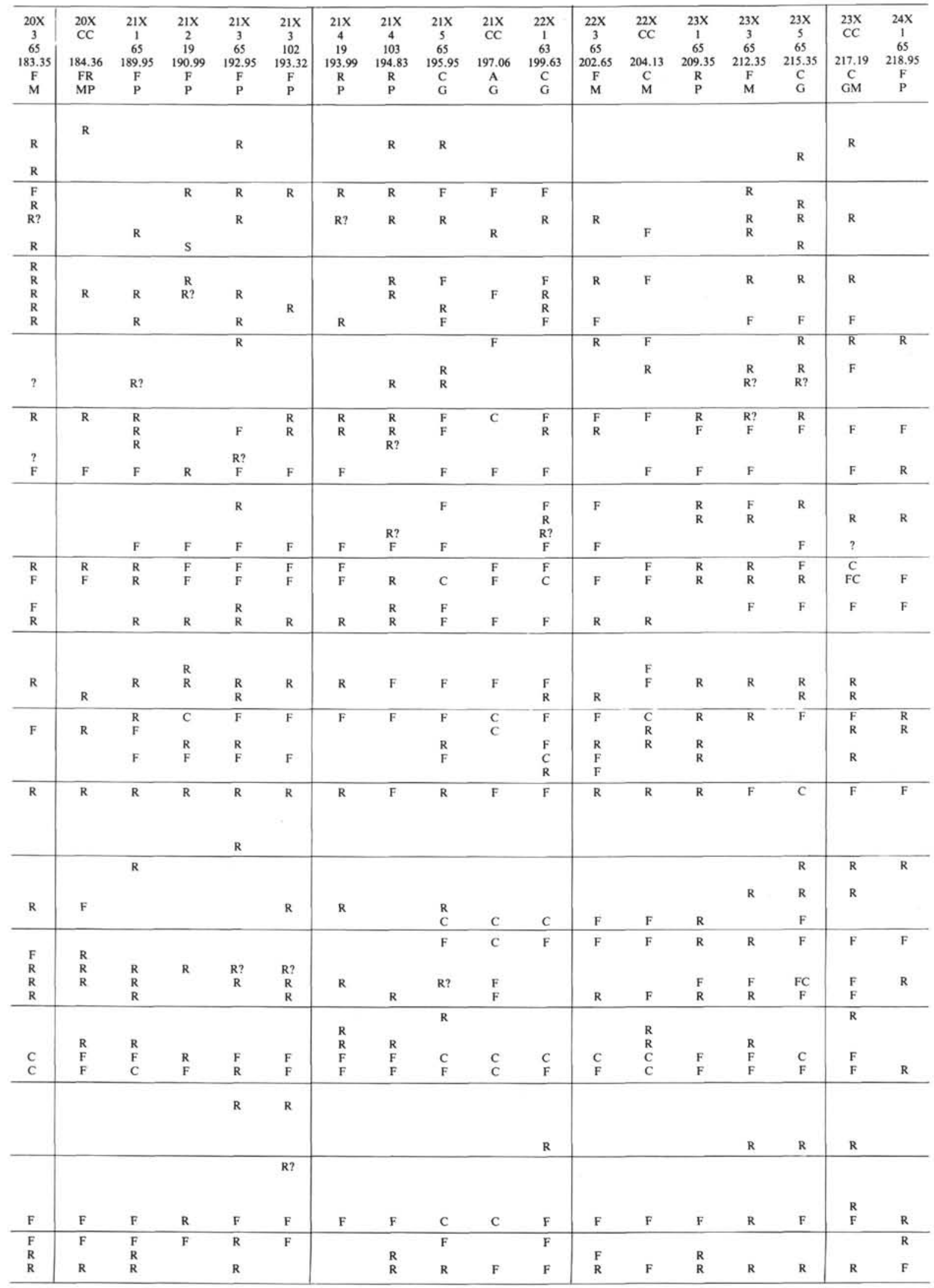




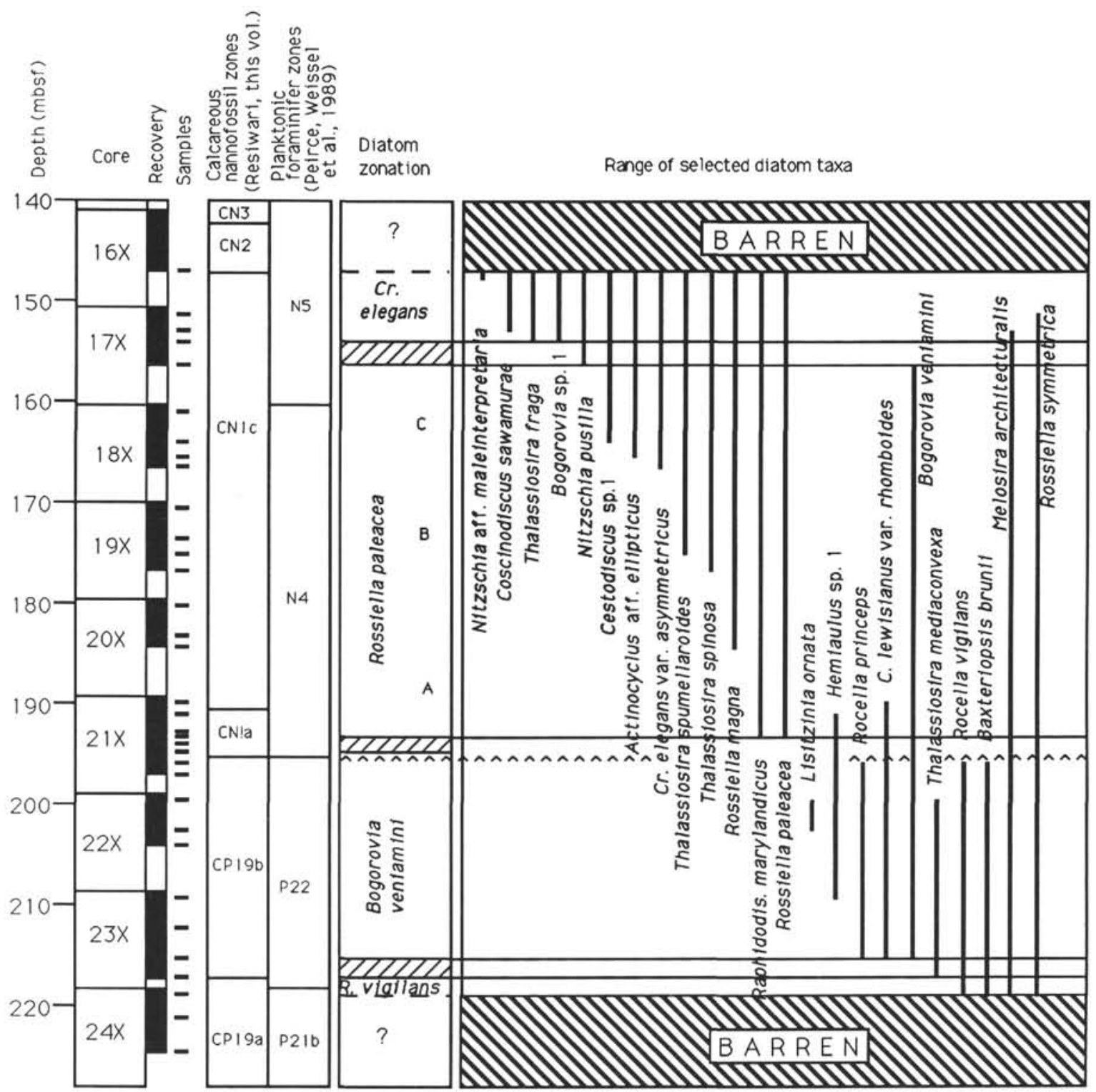

Figure 4. Diatom stratigraphy and ranges of selected diatom taxa for the Oligocene-Miocene diatomaceous interval at Hole 758A (Cores 121-758A-16X to 121-758A-24X). Calcareous nannofossil zonation after Resiwati (this volume) and planktonic foraminifers zonation after Gamson and Smit in Peirce, Weissel, et al. (1989). Diagonal lines represent intervals of uncertainty (inclined to right, interval between samples; inclined to left, dissolution). Undulating line indicates a hiatus.

paleacea in the tropical Pacific Ocean (Barron, 1983, 1985b). Considering the poor preservation of the diatoms in that section and the assignment of the section to the Miocene by calcareous nannofossils (Resiwati, this volume), it seems reasonable to suggest that Section 121-758A-21X-4 may correlate with the lower part of the Rossiella paleacea Zone, and that Rossiella paleacea has not been preserved in that interval. The absence of Rocella vigilans and Rocella princeps, resistant late Oligocene species, further distinguishes this interval from the interval lying immediately below Section 121-758A-21X-4.

Bogorovia veniamini Zone (24.0 to $26.5 \mathrm{Ma}$ )

The Bogorovia veniamini Zone is recognized between Sample $121-758 \mathrm{~A}-21 \mathrm{X}-5,65-67 \mathrm{~cm}$ (last occurrence of Rocella vigilans and Rocella princeps) and Sample 121-758A-23X-5, 65-67 cm (first occurrence of Bogorovia veniamini). The top of the zone is probably missing due to the presence of a hiatus around the Miocene/Oligocene boundary (see subsequent discussion).

Preservation is relatively good. Some characteristic taxa in this interval are Rocella vigilans, Rocella princeps, Synedra jouseana, Melosira architecturalis, Cestodiscus spp., Lisitzinia ornata, and Baxteriopsis brunii.

Rocella vigilans Zone (26.5 to $34 \mathrm{Ma}$ )

The Rocella vigilans Zone (Subzone B) is recognized in Samples 758A-23X-CC and 121-758A-24X-1, 65-67 cm, based on the absence of Bogorovia veniamini and co-occurrence of Rocella vigilans and Rossiella symmetrica. The sequence cannot be zoned beneath Sample 121-758A-24X-1, 65-67 cm, due to deterioration of the preservation of the assemblages. 


\section{Age and Duration of the Hiatus at the Miocene/Oligocene Boundary}

The absence of Rocella gelida at Site 758 and the abrupt change in opal preservation and species composition of the assemblages between Samples 121-758A-21X-4, 103-105 cm, and 121$758 \mathrm{~A}-21 \mathrm{X}-5,65-67 \mathrm{~cm}$, suggest the presence of a hiatus (or a very condensed section) between these two levels, around the Miocene/Oligocene boundary.

It is suggested from the above discussion that the oldest possible age for Sample 121-758A-21X-4, 103-105 cm, is $22.7 \mathrm{Ma}$ (age of the base of the Rossiella paleacea Zone).

Assuming that Rocella gelida has a similar range between the equatorial Pacific and the equatorial Indian Ocean, and that it was actually present at this site around the Miocene/Oligocene boundary, the minimum range for the missing interval at Site 758 would be between $22.7 \mathrm{Ma}$ and 24.5 Ma (range of Rocella gelida).

Furthermore, Sample 121-758A-21X-5, 65-67 contains common Rocella vigilans and Rocella princeps (but no Rocella gelida). Gombos (1983) records the highest common Rocella princeps at Site 516F in the South Atlantic in Sample 516F-11-3, $121-123 \mathrm{~cm}$ (268.3 mbsf), and the lowest Rocella gelida in Sample 516F-10-3, 100-102 cm (258.6 mbsf). This would correlate the last occurrence of Rocella princeps with the upper part of the reversed event between magnetic anomalies 7 and 7A (Berggren et al., 1983, p. 697), which is dated between 25.97 and 26.38 Ma (Berggren et al., 1985). This gives an approximate age of 26 $\mathrm{Ma}$ for the last occurrence of Rocella princeps at Site $516 \mathrm{~F}$ and suggests that the youngest possible age for Sample 121-758A$21 \mathrm{X}-5,65-67 \mathrm{~cm}$, could be about $26 \mathrm{Ma}$.

Strontium isotope analyses have been performed by Enriqueta Barrera at The University of Michigan on foraminifers from Samples 121-758A-21X-4, 103-105 cm, and -121-758A-21X-5, $65-67 \mathrm{~cm}$. The results are identical for these two samples with an age of 26.3 Ma (E. Barrera, pers. comm., 1990). Strontium isotopes confirm the age of ca. $26 \mathrm{Ma}$ estimated by diatom stratigraphy for Sample 121-758A-21X-5, 65-67 cm. However, an age of 26.3 Ma for Sample 121-758A-21X-4, 103-105 cm, is somewhat unexpected (perhaps E. Barrera dated a foraminifer reworked from the Oligocene). Nevertheless, strontium isotopes demonstrate the presence of sediments of $26 \mathrm{Ma}$ at the Miocene-Oligocene boundary as defined by both diatoms and calcareous nannofossils. (Resiwati, this volume, places the limit between Zones CN1 and CP19 between Sections 121-758A-21X-4 and $121-758 \mathrm{~A}-21 \mathrm{X}-5$.) This suggests that at least the upper part of the Oligocene ( 23.7 to $26 \mathrm{Ma}$ ) is missing and supports the evidence of a hiatus at the Miocene/Oligocene boundary.

In conclusion, at least the interval between 23.7 and $26 \mathrm{Ma}$, and very likely also the interval between 22.7 and $23.7 \mathrm{Ma}$, are missing or extremely condensed at Site $578 \mathrm{~A}$. This represents a hiatus that may be as long as $3.3 \mathrm{~m}$.y.

\section{Campanian}

Diatoms occur in the upper Campanian sediments of Site 758 (calcareous nannofossil Zones CC21-CC22) in a relatively short interval between Cores 121-758A-38X and -40X (approximately 352 to $377 \mathrm{mbsf}$ ). Poorly to moderately preserved diatom assemblages were found in the following samples: 121-758A-38X-CC; $-39 \mathrm{X}-\mathrm{CC} ;-40 \mathrm{X}-1,135-137 \mathrm{~cm} ;-40 \mathrm{X}-2,106-108 \mathrm{~cm}$; and $-40 \mathrm{X}-$ CC.

Despite the relatively poor preservation of the assemblages and the short range of the diatomaceous interval, the Campanian diatoms at Site 758 are of some interest, as the occurrence of
Cretaceous diatom sequences is very uncommon in the deep-sea record (see "Introduction" section, this chapter).

The observed taxa are listed in the taxonomic list (under the "Cretaceous Diatoms" section, this chapter). Species identifications are based on the works of Strelnikova (1974), Harwood (1988), and Hajós and Stradner (1975). Some characteristic species are illustrated in Table 5.

The assemblages are dominated by Stephanopyxis spp., Triceratium spp., Rhizosolenia cretacea, and Eunotogramma polymorphus. Other characteristic taxa are Gladius speciosus, Coscinodiscus morenoensis, and Trinacria pileolus.

\section{CONCLUSIONS}

The documentation of Quaternary to upper Pliocene, lower Miocene to upper Oligocene, and Campanian diatoms from Site 758 provides new information on the equatorial Indian Ocean. This study confirms the possibility of using the late Neogene low-latitude diatom zonation of Burckle (1972) in the tropical Indian Ocean. The early Miocene low-latitude diatom zonation of Barron (1983) is usable, although the lack of some stratigraphic markers prevents the separation of the different subzones of the Rossiella paleacea Zone. Similarly, Fenner's (1984) low-latitude zonation can be successfully used in the Oligocene. The Rocella gelida Zone (22.7 to $24.0 \mathrm{Ma}$ ) is not recognized at Site 758 . Although the species Rocella gelida may not have lived in the equatorial Indian Ocean, its absence, as well as results from strontium isotopes, suggest that a hiatus is present at the Miocene/Oligocene boundary.

Comparison of the age of late Pliocene to Quaternary stratigraphic events between the equatorial Indian Ocean, the equatorial Pacific, and the North Atlantic suggests synchroneity for some events and diachroneity for others. Future studies of additional cores should improve our knowledge of the evolution and migration history of diatom taxa. Climatic and paleoceanographic causes may be considered in the interpretation of such diachronisms.

\section{FLORAL REFERENCES AND TAXONOMIC NOTES}

Description of New Taxa and Transfer of Taxa

Craspedodiscus elegans Ehrenberg, 1844 var. asymmetricus Fourtanier, n. var.

$$
\text { (PI. 3, Figs. 6, 8) }
$$

Description. Craspedodiscus elegans var. asymmetricus has a large circular valve (specimens observed range in diameter between 80 and 130 $\mu \mathrm{m})$. The valves are concentrically undulated with a marginal ring and a central area raised to an approximately equal elevation. The undulation, however, is asymmetrical as the slopes demarcating the central and the peripheral areas are abrupt for one-half of the valve and gradual for the other half. The marginal ring occupies one-quarter of the radius in the abrupt-sloped portion of the valve and one-third of the radius in the other part. The central area occupies approximately one-quarter of the diameter of the valve. Hexagonal areolae are radially arranged and slightly increase in size from the center (about 4 areolae in $10 \mu \mathrm{m}$ ) to the periphery (about 3 areolae in $10 \mu \mathrm{m})$.

Remarks. Areolae pattern, and to some extent the concentric undulation of the valve, is similar to that of Craspedodiscus elegans s. str.; however, the asymmetry of the valve undulation distinguishes Craspedodiscus elegans var. asymmetricus from Craspedodiscus elegans var. elegans.

Occurrence. Craspedodiscus elegans var. asymmetricus occurs in small numbers in the lower Miocene of Site 758 (Craspedodiscus elegans Zone and Rossiella paleacea Zone).

Holotype. Slide number 216009 (California Academy of Sciences) (P1. 3, Fig. 6).

Type locality. Equatorial Indian Ocean, Sample 121-758A-18X-CC. 
Rossiella magna (Lohman) Fourtanier, n. comb.

$$
\text { (Pl. 4, Fig. 1) }
$$

Basionym. Cymatosira magna Lohman, 1974 (p. 351, pl. 5, fig. 16).

Original description. Lohman, 1974, p. 351: "Valve flat, lanceolate with subrostrate apices; valve surface covered completely with hexagonal areolae, 6-7 in $10 \mu \mathrm{m}$, in no order and without central space; margin with heavy striae, 7 in $10 \mu \mathrm{m}$; hexagonal areolae appear round with slight change of focus; valve symmetrical about longitudinal axis but somewhat asymmetrical about short axis. Length, 60-80 $\mu \mathrm{m}$; width, 16-20 $\mu \mathrm{m}$.

Remarks. This species lacks the marginal spines characteristic of the genus Cymatosira. In addition, this species, as described and illustrated by Lohman (1974), meets the characteristics of the genus Rossiella Desikachari et Maheshwari (1958, p. 28) by the elliptical elongated shape of the valves and the pore pattern. Transfer to genus Rossiella is therefore proposed.

This species closely resembles Rossiella paleacea (Grunow) Desikachari et Maheshwari (1958) for the shape of the valve and the size and arrangement of the areolae; however, it is typically longer and larger (60-80 $\mu \mathrm{m}$ in length for Cymatosira magna, according to Lohman [1974], 25-76 $\mu \mathrm{m}$ in length for Rossiella paleacea, according to Desikachari and Maheshwari [1958]).

Rossiella magna and Rossiella paleacea both occur in the lower Miocene sediments of Site 758. Rossiella magna, however, occurs slightly younger than does Rossiella paleacea, with a first occurrence in Sample 121-758A-20X-CC.

Although Rossiella magna has been commonly reported as Rossiella paleace $a$ in the literature, observation of material from the lower Miocene of Site 758 suggests that these two taxa can be differentiated and confirms the validity of Rossiella magna as a distinct species from Rossiella paleacea.

Synonyms. Rossiella paleacea (Grunow) Desikachary and Maheshwari (robust form) (Barron, 1983, pl. 5, fig. 6); Coscinodiscus paleaceus (Grunow) Rattray (Jousé, 1973, pl. 4, fig. 4).

\section{Species List and Taxonomic Notes}

All diatom taxa considered in this study are enumerated below. Several species are illustrated in Plates 1-5. For species not illustrated in this report, reference to illustrations is given. Comments are included for several species.

\section{Cenozoic Diatoms}

Actinocyclus curvatulus Janisch 1878 in Schmidt et al., 1874-; Barron (1980), pl. 1 , fig. 1

Actinocyclus ellipticus Grunow in Van Heurck, 1883; Barron (1980), pl. 1 , fig. 3,6 .

Actinocyclus ellipticus aff. ellipticus Grunow in Van Heurck, 1883 (P1. 2, Fig. 1).

Actinocyclus ellipticus var. elongatus (Grunow in Van Heurck 1883) Kolbe 1954; Schrader (1974), pl. 17, fig. 1.

Actinocyclus octonarius Ehrenberg 1838.

Actinoptychus senarius (Ehrenberg 1838?) Ehrenberg, 1843; Schrader (1973), pl. 22, fig. 12.

Actinoptychus spp.

Asterolampra aff. acutiloba Forti, 1912.

Remarks. The rare specimens of this taxon observed in the upper Oligocene at Site 758 are very similar to the specimen figured in Gombos and Ciesielski (1983, pl. 1, fig. 8) as Asterolampra acutiloba from the lower Oligocene of DSDP Site 511. However, they differ in shape from Asterolampra acutiloba s. str. and are morphologically situated between Asterolampra acutiloba Forti (see Schrader, 1974 pl. 9, figs. 7, 8, for a good illustration) and Asterolampra praeacutiloba Fenner in Schrader and Fenner 1976 (Schrader and Fenner, 1976 pl. 28, figs. 9-11).

Asterolampra insignis Schmidt, 1889 in Schmidt et al., 1874 - (group); Schrader and Fenner (1976), pl. 21, fig. 15

Asterolampra marylandica Ehrenberg, 1844; Schrader (1974) pl. 8, fig. 2.

Asterolampra aff. punctifera (Grove, 1896) Hanna, 1927; Gombos and Ciesielski (1983), pl. 2, figs. 4-8.

Asterolampra spp.

Asteromphalus arachne (Brébisson, 1857) Ralfs in Pritchard, 1861; Schrader (1974), pl. 9, fig. 11.
Asteromphalus aff. hepactis (Brébisson, 1857) Ralfs in Pritchard, 1861; Schrader (1974), pl. 8, fig. 1.

Asteromphalus oligocenicus Schrader et Fenner, 1976; Schrader and Fenner (1976), pl. 21, figs. 13, 14.

Asteromphalus spp.

Azpeitia africana (Janisch, 1878 in Schmidt et al., 1874-) Fryxell et Watkins in Fryxell et al., 1986; Fryxell et al. (1986), figs. 22, 23.

Azpeitia endoi (Kanaya, 1959) Sims et Fryxell in Fryxell et al., 1986; Schrader (1974), pl. 18, figs. 5-7.

Azpeitia nodulifer (Schmidt, 1878 in Schmidt et al., 1874-) Fryxell et Sims in Fryxell et al., 1986; Fryxell et al. (1986), figs. 17, 18.

Azpeitia oligocenica (Jousé, 1973) Sims in Sims et al., 1989 (Pl. 4, Fig. 7).

Azpeitia praenodulifera (Barron, 1983) Sims et Fryxell in Sims et al., 1989; Sims et al. (1989), pl. 1, figs. 3-13.

Azpeitia salisburyana (Lohman, 1948) Sims in Sims et al., 1989; Sims et al. (1989), pl. 2, figs. 4-6.

Azpeitia tabularis (Grunow, 1884) Fryxell et Sims in Fryxell et al., 1986; Fryxell et al. (1986), figs. 14, 15.

Azpeitia spp.

Bacteriastrum sp.

Baxteriopsis brunii (Van Heurck, 1893) Karsten, 1928 (Pl. 3, Fig. 1).

Bogorovia veniamini Jousé, 1973 (Pl. 4, Fig. 3).

Bogorovia sp. 1 (fine forms) (Pl. 4, Fig. 6).

Bogorovia intermediate between Bogorovia veniamini and Rossiella symmetrica (Pl. 4, Fig. 2).

Cestodiscus japonicus Cleve, 1877 (P1. 1, Fig. 7?); Radionova (1987), pl. 4 , figs. 5-7.

Cestodiscus kugleri Lohman, 1974 (Pl. 1, Fig. 6).

Cestodiscus ovalis Greville, 1865 (Pl. 2, Fig. 2).

Cestodiscus pulchellus Greville, 1866; Radionova (1987), pl. 2, figs. 3-5.

Cestodiscus stokesianus Greville, 1866 (Pl. 2, Fig. 4?); Radionova (1987), pl. 5, figs. 3, 4 .

Cestodiscus trochus Castracane, 1886 (Pl. 1, Fig. 4); Radionova (1987), pl. 3 , fig. 2 .

Cestodiscus sp. 1 (Pl. 1, Fig. 2).

Cestodiscus sp. 3 (Pl. 1, Fig. 3).

Cestodiscus sp. 4 (P1. 1, Fig. 1).

Cestodiscus spp. (Pl. 1, Fig. 5; Pl. 2, Fig. 3; and other species not illustrated).

Coscinodiscus lewisianus Greville, 1866; Barron (1983), pl. 1, fig. 5.

Coscinodiscus lewisianus var. rhomboides Barron, 1985; Barron (1983), pl. 1, fig. 8 (as Coscinodiscus lewisianus var. similis); Barron (1985b), pl. 7 , figs. 2,3 .

Coscinodiscus marginatus Ehrenberg, 1841.

Coscinodiscus radiatus Ehrenberg, 1839.

Coscinodiscus rhombicus Castracane, 1886; Barron (1983), pl. 1, figs. $10,11$.

Coscinodiscus sawamurae Akiba, 1980 (Pl. 2, Fig. 5); Akiba (1980), pl. 4, figs. 44-46; Fourtanier and Macharé (1988), pl. 2, figs. 28, 29.

Coscinodiscus spp.

Craspedodiscus barronii Bukry, 1984 (Pl. 3, Fig. 7).

Original reference. Bukry (1984), p. 464, pl. 3, figs. 1-5.

Remarks. Craspedodiscus barronii is distinguished from Craspedodiscus coscinodiscus Ehrenberg by "regular radial lines of areolae from center point to margin, by secondary spiral, and by the absence of a sharp demarcation boundary between central and peripheral areas."

Synonyms. Craspedodiscus aff. coscinodiscus Ehrenberg (Jousé, 1977, pl. 56, fig. 5); Craspedodiscus coscinodiscus s. ampl. (Barron, 1985b, pl. 2, fig. 3); Craspedodiscus coscinodiscus Ehr. var.? (Jousé, 1973, pl. 3, fig. 1).

Craspedodiscus elegans var. elegans Ehrenberg, 1844; Barron (1985b), pl. 2 , fig. 2 .

Craspedodiscus elegans var. asymmetricus Fourtanier n. var. (described above; Pl. 3, Figs. 6, 8).

Cyclotella spp.

Dimerogramma sp. aff. D. fulvum (Gregory, 1857) Ralfs in Pritchard, 1861; Schrader and Fenner (1976), pl. 7. figs. 19, 20.

Ethmodiscus rex (Wallich in Rattray, 1890) Hendey in Wiseman and Hendey, 1953; Barron (1980), pl. 10, figs. 3, 6.

Grammatophora spp.

Hemiaulus longicornis Greville, 1865 (P1. 3, Fig. 4).

Hemiaulus subacutus Grunow, 1884 (Pl. 3, Fig. 2). 
Hemiaulus sp. 1 (Pl. 3, Fig. 3).

Hemiaulus spp.

Hemidiscus cuneiformis Wallich, 1860; Schrader (1974), pl. 19, figs. 22-25.

Hyalodiscus spp.

Lisitzinia ornata Jousé, 1978 (Pl. 3, Fig. 5).

Melosira architecturalis Brun, 1892 in Schmidt et al., 1874- (P1. 2, Fig. 7).

Nitzschia fossilis (Frenguelli, 1949) Kanaya in Kanaya and Koizumi, 1970; Barron (1980), pl. 6, figs. 4-7.

Nitzschia interrupta (Reichelt in Kuntze, 1898) Hustedt, 1927; Schrader (1974), pl. 15, figs. 1-7.

Nitzschia aff. maleinterpretaria Schrader, 1976 (P1. 4, Fig. 11)

Nitzschia marina Grunow in Cleve and Grunow, 1880; Schrader (1974), pl. 5 , figs. 1,2 .

Nitzschia pusilla Schrader, 1976; Schrader, 1976, pl. 2, fig. 20.

Nitzschia reinholdii Kanaya ex Barron et Baldauf, 1986; Barron (1980) pl. 6, figs. 10,11.

Nitzschia aff. sicula (Castracane) Hustedt, 1958; Akiba (1986), pl. 24, fig. 4.

Nitzschia spp.

Paralia sulcata (Ehrenberg, 1838) Cleve, 1873; Schrader (1973), pl. 20, fig. 9 (as Melosira sulcata).

Pseudoeunotia doliolus (Wallich, 1860) Grunow in Van Heurck, 1881; Barron (1980), pl. 6, figs. 1-3.

Raphidodiscus marylandicus Christian, 1887 (Pl. 2, Fig. 6).

Rhaphoneis sp.

Rhaphoneis sp. aff. fossile (Grunow in Cleve and Möller, 1877-82) Andrews, 1978.

Rhizosolenia alata Brightwell, 1858; Jousé (1977), pl. 7, fig. 6.

Rhizosolenia aff. alata Brightwell, 1858 (Pl. 4, Fig. 10).

Rhizosolenia bergonii H. Péragallo, 1892; Schrader (1974), pl. 9, fig. 4.

Rhizosolenia firma Karsten, 1907; Schrader (1974), pl. 6, fig. 6.

Rhizosolenia praebergonii var. praebergonii Mukhina, 1965; Barron (1980), pl. 7, figs. 3, 4.

Rhizosolenia praebergonii var. robusta Burckle et Trainer, 1979; Barron (1980), pl. 7, figs. 5-8.

Rhizosolenia styliformis Brightwell, 1858; Schrader (1973), pl. 10, figs. $18,19$.

Rocella princeps (Jousé, 1973) Fenner, 1984 (Pl. 4, Fig. 8).

Rocella vigilans Fenner, 1984 (Pl. 4, Fig. 9).

Roperia tesselata (Roper, 1858) Grunow in Van Heurck, 1883; Schrader (1974), pl. 21, figs. 9-12.

Rossiella paleacea (Grunow in Van Heurck, 1883) Desikachari et Maheshwari, 1958 (PI. 4, Fig. 4).

Rossiella magna (Lohman, 1974) Fourtanier n. comb. (Pl. 4, Fig. 1).

Rossiella symmetrica Fenner, 1984 (Pl. 4, Fig. 5).

Rouxia quadrangula Jousé, 1973 (Pl. 4, Fig. 12).

Sceptroneis sp.

Stephanopyxis turris (Greville et Arnott, 1857) Ralfs in Pritchard, 1861; Jousé (1977), pl. 16, figs. 3-5.

Stephanopyxis sp.

Synedra jouseana Sheshukova-Poretzkaya, 1962; Barron (1985b), pl. 8, fig. 17.

Stellarima spp.

Thalassionema bacillaris (Heiden et Kolbe, 1928) Kolbe, 1955.

Thalassionema nitzschioides (Grunow, 1862) Van Heurck, 1896.

Thalassionema nitzschioides var. parva Heiden et Kolbe, 1928.

Thalassionema spp.

Thalassiosira bukryi? Barron, 1983.

Remarks. Specimens related to Thalassiosira bukryi occur in the lower Miocene of Site 758. Their pore pattern is similar to those of $T$. bukryi (Barron, 1983, pl. 4, figs. 1, 2); however, they seem to have a central process similar to the central labiate process typical of the genus Azpeitia. SEM studies are necessary to clarify the taxonomic position of the specimens observed at Site 758.

Thalassiosira convexa var, aspinosa Schrader, 1974; Schrader, 1974, pl. 2 , figs. $13 \mathrm{~A}-21$.

Thalassiosira eccentrica (group) (Ehrenberg, 1841) Cleve, 1903 (1904).

Thalassiosira fraga Schrader in Schrader and Fenner, 1976 (Pl. 2, Fig. 8).

Thalassiosira aff. fraga Schrader (fine).
Remarks. These forms are related to the species Thalassiosira fraga but are typically more finely areolated. They occur in the lower Miocene of Site 758.

Thalassiosira lacustris (group) (Grunow in Cleve et Grunow, 1880) Hasle in Hasle et Fryxell, 1977; Barron (1980), pl. 5, figs. 8, 9 (as Coscinodiscus plicatus group).

Thalassiosira leptopus (Grunow in Van Heurck, 1883) Hasle et Fryxell, 1977.

Thalassiosira lineata (group) Jousé, 1968.

Thalassiosira mediaconvexa Schrader in Schrader et Fenner, 1976 (Pl. 2, Fig. 11).

Thalassiosira oestrupii (Ostenfeld, 1900) Proshkina-Lavrenko, 1965; Schrader, 1974, pl. 1, figs. 4, 5.

Thalassiosira spinosa Schrader, 1976 (Pl. 2, Figs. 9, 10?).

Thalassiosira spumellaroides Schrader, 1976 (Pl. 2, Fig. 12).

Thalassiosira spp.

Thalassiothrix longissima Cleve et Grunow in Cleve and Möller, 1878.

Thalassiothrix spp.

Triceratium cinamomeum Greville, 1863; Barron (1980), pl. 10, fig. 10.

\section{Cretaceous Diatoms}

Coscinodiscus morenoensis Hanna, 1927 (PI. 5, Fig. 13); Hajós and Stradner (1975), pl. 3, figs. 6, 7 .

Eunotogramma polymorphus Strelnikova, 1965 (Pl. 5, Figs. 1, 2); Strelnikova (1974), pl. 52, figs. 10, 11.

Eunotogramma sp. (Pl. 5, Fig. 3).

Remarks. Taxon identical to Eunotogramma polymorphus Strelnikova of Strelnikova (1974), pl. 52, figs. 8, 9.

Gladius speciosus Schulz, 1935 (P1. 5, Figs. 9, 10).

Hemiaulus sp. 1 (Pl. 5, Fig. 14).

Hemiaulus sp. 2 (Pl. 5, Fig. 15).

Hemiaulus spp.

Pterotheca aculeifera (Grunow in Van Heurck, 1883) Van Heurck, 1896; Harwood (1988), figs. 18.3-4.

Pyxidicula minuta Grunow, 1884?; Hajós and Stradner (1975), pl. 1, figs. $16-18$.

Rhizosolenia cretacea Hajós et Stradner 1975 (Pl. 5, Fig. 12).

Stephanopyxis reticulata Long, Fuge et Smith, 1946 (PI. 5, Fig. 11); Strelnikova (1974), pl. 5, figs. 7-13.

Stephanopyxis schulzii Steinecke, 1935 (Pl. 5, Fig. 8); Strelnikova (1974), pl. 4, figs. 1-3, 8 .

Stephanopyxis turris (Greville et Arnott, 1857) Ralfs in Pritchard, 1861; Harwood (1988), figs. 19.26-27.

Stephanopyxis spp.

Triceratium schulzii Jousé, 1949? (Pl. 5, Fig. 6)

Remarks. Taxa similar to Triceratium schulzii Jousé illustrated in Strelnikova (1974), pl. 29, figs. 1-7.

Triceratium sp. 1 (P1. 5, Figs. 4, 5).

Triceratium spp.

Trinacria pileolus (Ehrenberg, 1844) Grunow, 1884 (Pl. 5, Fig. 7); Harwood (1988), fig. 21.20.

\section{Other Taxa (Cenozoic)}

Melosira granulata s. ampl. (freshwater diatom) (Ehrenberg, 1843) Ralfs in Pritchard, 1861.

Mesocena elliptica (silicoflagellate) Ehrenberg, 1872 ex Haeckel, 1887; Baldauf (1987), pl. 3, fig. 1; pl. 6, fig. 11.

Macrora stella (incertae sedis) (Azpeitia, 1911) Hanna, 1932; Baldauf (1987), pl. 4, fig. 4

\section{ACKNOWLEDGMENTS}

I thank ODP France and INSU for providing financial support, and the entire Leg 121 staff for making Leg 121 a successful cruise. I am grateful to the USGS for providing space and facilities, and to Karen Hansen and Lisa Meng for helping in sample preparation. The manuscript benefitted from the reviews of Lloyd Burckle and Jack Baldauf; John Barron also provided helpful comments. Enriqueta Barrera (University of Michigan) gener- 
ously provided strontium isotope analysis of two samples from Site 758.

\section{REFERENCES}

Akiba, F., 1980. A lower Miocene diatom flora from the Boso Peninsula, Japan, and the resting spore formation of an extinct diatom, Kisseleviella carina Sheshukova-Poretzkaya. Bull. Tech. Lab., JAPEX, 23:81-94.

1986. Middle Miocene to Quaternary diatom biostratigraphy in the Nankai Trough and Japan Trench, and modified lower Miocene through Quaternary diatom zones for middle-to-high latitudes of the North Pacific. In Kagami, H., Karig, D. E., Coulbourn, W. T., et al., Init. Repts. DSDP, 87: Washington (U.S. Govt. Printing Office), 393-481.

Baldauf, J. G., 1987. Diatom biostratigraphy of the middle- and high-latitude north Atlantic Ocean, Deep Sea Drilling Project Leg 94. In Ruddiman, W. F., Kidd, R. B., Thomas, E., et al., Init. Repts. DSDP, 94 (Pt. 2): Washington (U.S. Govt. Printing Office), 729-762.

Barron, J. A., 1980. Upper Pliocene and Quaternary diatom biostratigraphy of Deep Sea Drilling Project Leg 54, tropical easter Pacific. In Rosendahl, B. R., Hekinian, R., et al., Init. Repts. DSDP, 54: Washington (U.S. Govt. Printing Office), 455-485.

1983. Latest Oligocene through early middle Miocene diatom biostratigraphy of the eastern tropical Pacific. Mar. Micropaleontol., $7: 487-515$

1985a. Diatom biostratigraphy of the CESAR 6 core, Alpha Ridge. In Jackson, H. R., Mudie, P. J., and Blasco, S. M. (Eds.), Initial Geological Report on CESAR: The Canadian Expedition to Study the Alpha Ridge, Arctic Ocean. Geol. Surv. Can., Paper 84-22:137-148. 1985b. Late Eocene to Holocene diatom biostratigraphy of the equatorial Pacific Ocean, Deep Sea Drilling Project Leg 85. In Mayer, L., Theyer, F., Thomas, E., et al., Init. Repts. DSDP, 85: Washington (U.S. Govt. Printing Office), 413-456.

1985c. Miocene to Holocene planktonic diatoms In Bolli, H. M., Saunders, J. B., and Perch-Nielsen, K. (Eds.), Plankton Stratigraphy: Cambridge (Cambridge Univ. Press), 763-809.

in press. Neogene diatom datum levels in the equatorial and North Pacific. In Saito, T., and Ishizaki, K. (Eds.), The Centenary of Japanese Micropaleontology: Tokyo (Univ. Tokyo Press).

Berggren, W. A., Aubry, M.-P., and Hamilton, N., 1983. Neogene magnetostratigraphy of Deep Sea Drilling Project Site 516 (Rio Grande Rise, South Atlantic). In Barker, P. F., Carlson, R. L., Johnson, D. A., et al., Init. Repts. DSDP , 72: Washington (U.S. Govt. Printing Office), 675-713.

Berggren, W. A., Kent, D. V., Flynn, J. J., and Van Couvering, J. A., 1985. Cenozoic geochronology. Geol. Soc. Am. Bull., 96:1407-1418.

Bukry, D., 1974. Coccolith and silicoflagellate stratigraphy, eastern Indian Ocean, Deep Sea Drilling Project, Leg 22. In von der Borch, C. C., Slater, J. G., et al., Init. Repts. DSDP, 22: Washington (U.S. Govt. Printing Office), 601-607.

1984. Neogene silicoflagellates from Deep Sea Drilling Project Site 543, western tropical Atlantic Ocean. In Biju-Duval, B., Moore, J. C., et al., Init. Repts. DSDP, 78A: Washington (U.S. Govt. Printing Office), 463-468.

Burckle, L. H., 1972. Late Cenozoic planktonic diatom zones from the eastern equatorial Pacific. Nova Hedwigia Beih., 39:217-246.

1977. Pliocene and Pleistocene diatom datum levels from the equatorial Pacific. Quat. Res., 7:330-340.

1989. Distribution of diatoms in sediments of the northern Indian Ocean: relationship to physical oceanography. Mar. Micropaleontol., 15:53-65.

Burckle, L. H., and Opdyke, N. D., 1977. Late Neogene diatom correlations in the circum-Pacific. Proc. First Int. Congr. Pacific Neogene Stratigr. (Tokyo, 1976): Tokyo (Kaiyo Shupan Co. Ltd.) 255-284.

Desikachary, T. V., and Maheshwari, C. L., 1958. Fossil diatoms from Colebrook Island. J. Indian Bot. Soc., 38:27-41.

Fenner, J., 1982. Diatoms in the Eocene and Oligocene sediments off NW Africa: their stratigraphic and paleogeographic occurrences [Ph.D. dissert.]. Univ. of Kiel, Federal Republic of Germany.

1984. Eocene-Oligocene planktic diatom stratigraphy in the low latitudes and the high southern latitudes. Micropaleontology, 30:319-342
Fourtanier, E., and Macharé, J., 1988. Late Eocene to Pliocene marine diatoms from Peru. In Round, F. E. (Ed.), Proc. 9 th Int. Diatom Symp.: Bristol, UK, and Koenigstein, FRG (Biopress Ltd. and Koeltz Scientific Books), 151-163.

Fryxell, G. A., Sims, P. A., and Watkins, T. P., 1986: Azpeitia (Bacillariophyceae): related genera and promorphology. Syst. Bot. Monographs, 13:1-74.

Gombos, A. M., Jr., 1983. Survey of diatoms in the upper Oligocene and lower Miocene in Holes 515B and 516F. In Barker, P. F., Carlson, R. L., Johnson, D. A., et al., Init. Repts. DSDP, 72: Washington (U.S. Govt. Printing Office), 793-804.

Gombos, A. M., Jr., and Ciesielski, P. F., 1983. Late Eocene to early Miocene diatoms from the southwest Atlantic. In Ludwig, W. J., Krasheninnikov, V. A., et al., Init. Repts. DSDP, 71 (Pt. 2): Washington (U.S. Govt. Printing Office), 583-684.

Hajós, M., and Stradner, H., 1975. Late Cretaceous archaeomona-daceae, diatomaceae, and silicoflagellatae from the South Pacific Ocean, Deep Sea Drilling Project, Leg 29, Site 275. In Kennett, J. P., Houtz, R. E., et al., Init.Repts. DSDP, 29: Washington (U.S. Govt. Printing Office), 913-1009.

Harwood, D. M., 1988. Upper Cretaceous and lower Paleocene diatom and silicoflagellate biostratigraphy of Seymour Island, eastern Antarctic Peninsula. In Feldman, R., and Woodburne, M. O. (Eds.), Geology and Paleontology of Seymour Island. Geol. Soc. Am. Mem., 169:55-129.

Jousé, A. P., 1974. Diatoms in the Oligocene-Miocene biostratigraphic zones of the tropical areas of the Pacific Ocean. Nova Hedwigia Beih., 45:333-364.

1977. Atlas of Microorganisms in Bottom Sediments of the Oceans (Diatoms, Radiolaria, Silicoflagellates and Coccoliths): Moscow (NAUKA). [In Russian].

Jousé, A. P., and Kazarina, G. H., 1974. Pleistocene diatoms from Site 262, Leg 27, DSDP. In Veevers, J. J., Heirtzler, J. R., et al., Init. Repts. DSDP, 27: Washington (U.S. Govt. Printing Office), 925-946.

Kazarina, G. Kh., 1975. Diatom zones in the sediments of the eastern tropical region of the Indian Ocean. Okeanol. Akad. Nauk USSR, 15:1073-1078. [In Russian].

Kim, W. H., and Barron, J. A., 1986. Diatom biostratigraphy of the upper Oligocene to lowermost Miocene San Gregorio Formation, Baja California Sur, Mexico. Diatom Res., 1:169-187.

Koizumi, I., and Yanagisawa, Y., 1990. Evolutionary change in diatom morphology - an example from Nitzschia fossilis to Pseudoeunotia doliolus. Trans. Proc. Palaeont. Soc. Japan, N.S., 157:347-359.

Leg 121 Shipboard Scientific Party, 1988. A tale of two ridges. Nature, 335:593-594.

Lohman, K. E., 1974. Lower Miocene marine diatoms from Trinidad. Verhandl. Naturf. Ges. Basel, 84:326-360.

Mikkelsen, N., 1990. Cenozoic diatom biostratigraphy and paleoceanography of the western equatorial Indian Ocean. In Duncan, R. A., Backman, J., Peterson, L. C., et al., 1990. Proc. ODP, Sci. Results, 115: College Station, TX (Ocean Drilling Program), 411-432.

Oreshkhina, T. V., and Radionova, E. P., 1987. Korreliatsia zonalikh diatomovikh chak miotsena ekvatorialno-tropicheskikh i umerennikh chirot tikhogo okeana. Seria Geologisheskaia, 2:59-72.

Peirce, J., Weissel, J., et al., 1989. Proc. ODP, Init. Repts., 121: College Station, TX (Ocean Drilling Program).

Radionova, E. P., 1987. Diatom morphology of the genus Cestodiscus from the lower-middle Miocene deposits of the Pacific tropical zone. Questions of Micropaleontol., Moscow (NAUKA), 29:141-154. [In Russian].

Saito, T., and Burckle, L. H., 1977. Occurrence of silicoflagellate Mesocena elliptica: further evidence on the age of the Wakimoto Formation, Oga Peninsula, Japan and the recognition of the Jaramillo Event. Chishitsugaku Zasshi, 83:181-186.

Schrader, H.-J., 1973. Cenozoic diatoms from the Northeast Pacific, Leg 18. In Kulm, L. D., von Huene, R., et al., Init. Repts. DSDP, 18: Washington (U.S. Govt. Printing Office), 673-797.

1974. Cenozoic marine planktonic diatom stratigraphy of the tropical Indian Ocean. In Fisher, R. L., Bunce, E. T., et al., Init. Repts. DSDP, 24: Washington (U.S. Govt. Printing Office), 887-967. 1976. Cenozoic planktonic diatom biostratigraphy of the Southern Pacific Ocean. In Hollister, C. D., Craddock, C., et al., Init. Repts. DSDP, 35: Washington (U.S. Govt. Printing Office), 605-672. 
1978. Diatoms in DSDP Leg 41 sites. In Lancelot, Y., Seibold, E., et al., Init. Repts. DSDP, 41: Washington (U.S. Govt. Printing Office), 791-812.

Schrader, H.-J., and Fenner, J., 1976. Norwegian Sea Cenozoic diatom biostratigraphy and taxonomy. In Talwani, M., Udintsev, G., et al., Init. Repts. DSDP, 38: Washington (U.S. Govt. Printing Office), 921-1099.

Sims, P. A., Fryxell, G. A., and Baldauf, J. G., 1989. Critical examination of the diatom genus Azpeitia: species useful as stratigraphic markers for the Oligocene/Miocene Epochs. Micropaleontology, 35:293-307.
Strelnikova, N. I., 1974. Diatomei Posdnego Mela (Zapadnaya Sibiri). Akad. Nauk SSSR, 8.

Date of initial receipt: 2 March 1990

Date of acceptance: 4 October 1990

Ms 121B-137 


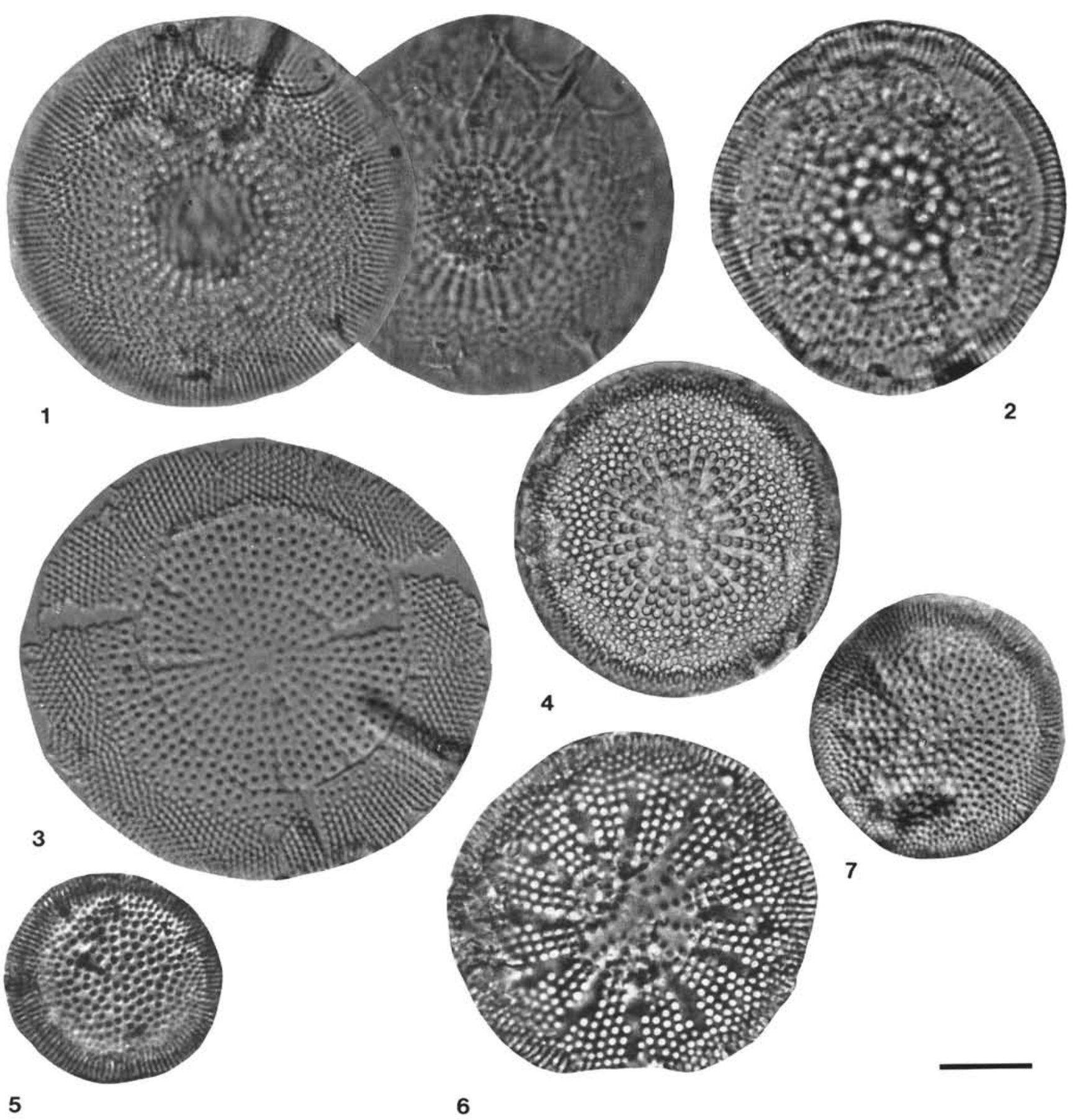

Plate 1. Oligocene-Miocene diatoms at Site 758 (all figures 1500x; scale bar $=10 \mu \mathrm{m}$ ). 1. Cestodiscus sp. 4, Sample 121-758A-23X-1, 65-67 cm, high and low focus. 2. Cestodiscus sp. 1, Sample 121-758A-18X-1, 65-67 cm. 3. Cestodiscus sp. 3, Sample 121-758A-18X-1, 65-67 cm. 4. Cestodiscus trochus Castracane, Sample 121-758A-20X-CC. 5. Cestodiscus sp., Sample 121-758A-21X-4, 19-21 cm. 6. Cestodiscus kugleri Lohman, Sample 121-758A-19X-1, 65-67 cm. 7. Cestodiscus japonicus Cleve?, Sample 121-758A-21X-CC. 


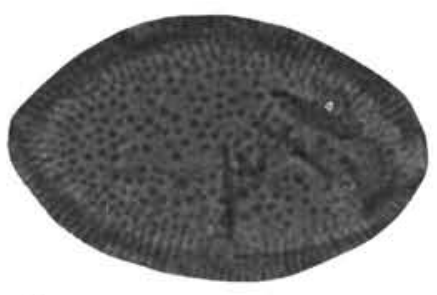

1

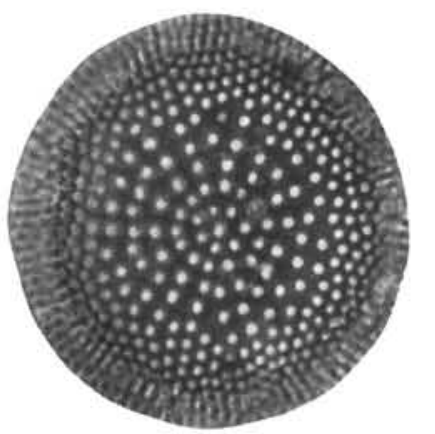

4

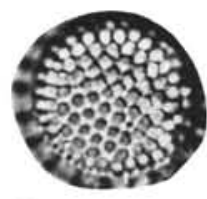

8

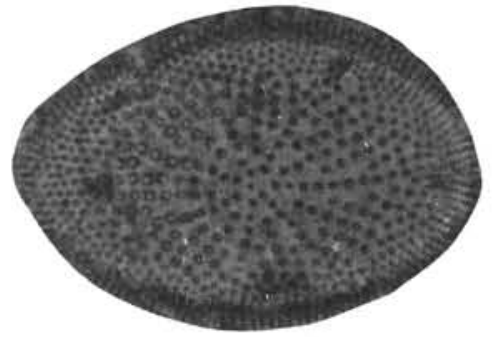

2

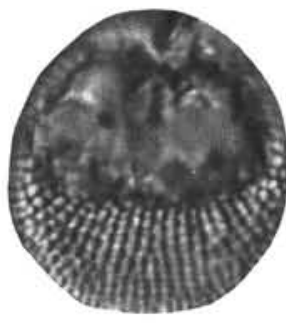

5

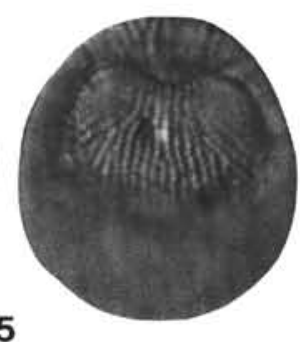

3
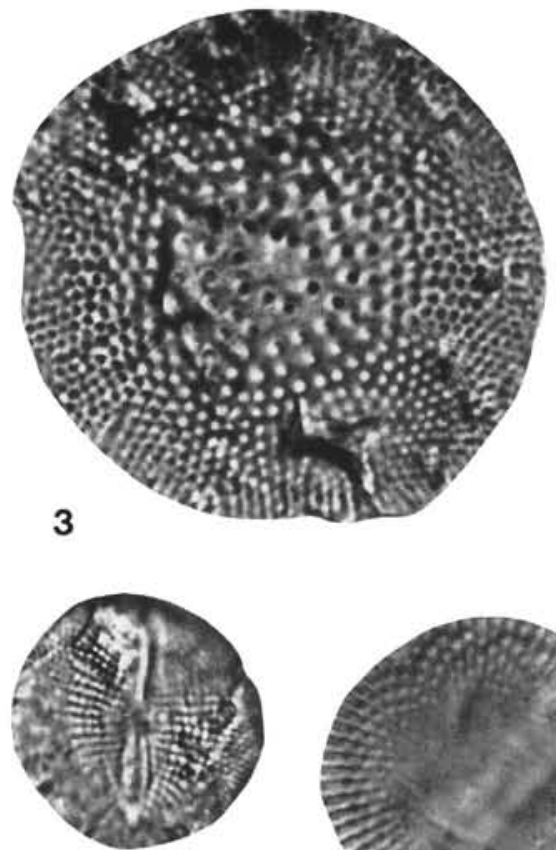

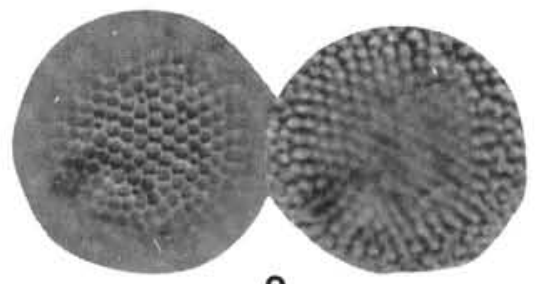

9
6

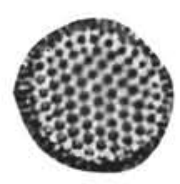

10
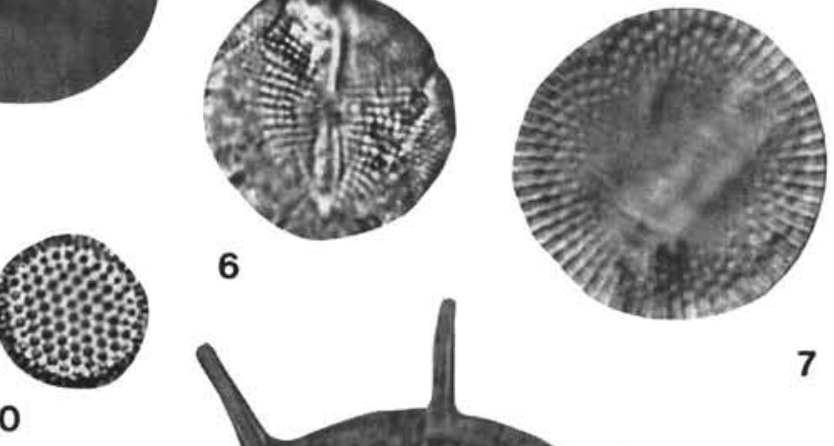

7
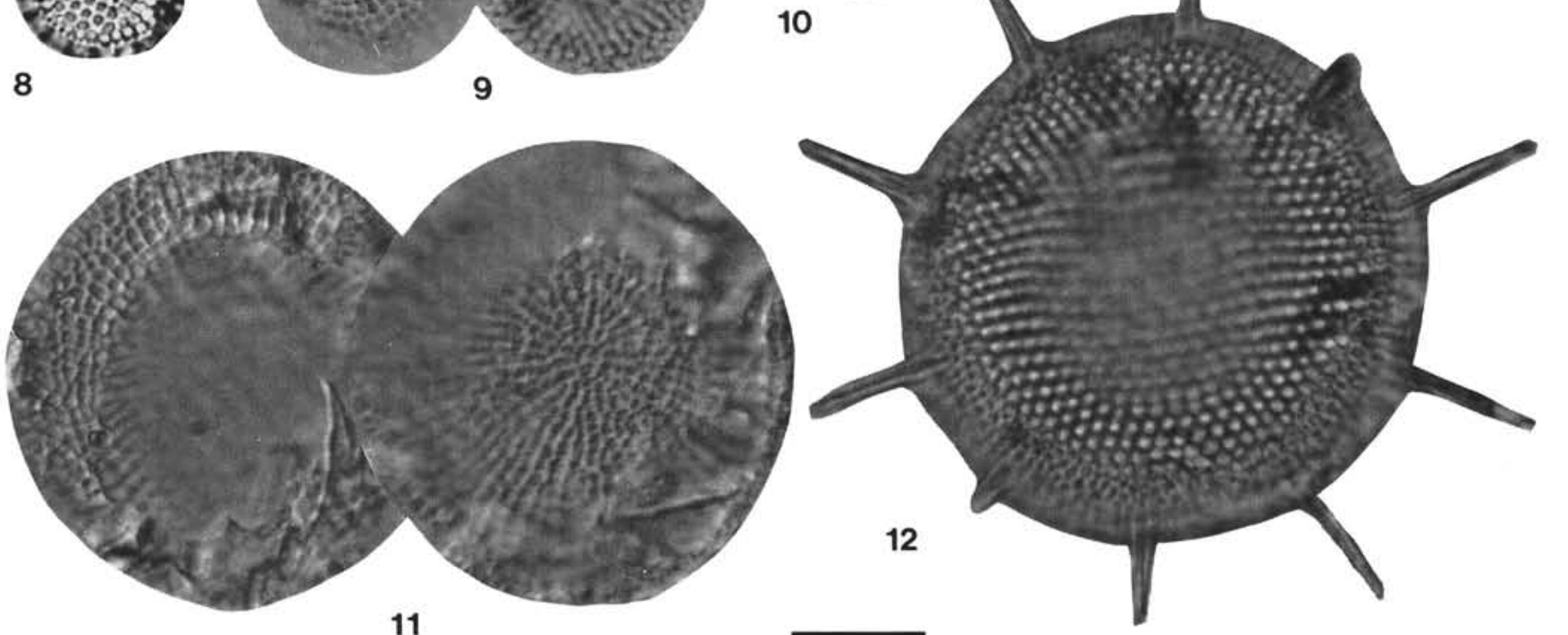

2

11

Plate 2. Oligocene-Miocene diatoms at Site 758 (all figures 1500x; scale bar $=10 \mu \mathrm{m}$ ). 1. Actinocyclus aff. ellipticus Grunow, Sample 121-758A-18X-4, 65-67 $\mathrm{cm}$. 2. Cestodiscus ovalis Greville, Sample 121-758A-18X-4, 65-67 cm. 3. Cestodiscus sp., Sample 121-758A-22X-1, 63-65 cm. 4. Cestodiscus stokesianus Greville?, Sample 121-758A-21X-3, 65-67 cm. 5. Coscinodiscus sawamurae Akiba, Sample 121-758A-17X-1, 65-67 cm, high and low focus. 6. Raphidodiscus marylandicus Christian, Sample 121-758A-18X-1, 65-67 cm. 7. Melosira architecturalis Brun, Sample 121-758A-22X-1, 63-65 cm. 8. Thalassiosira fraga Schrader, Sample 121-758A-17X-1, 65-67 cm. 9. Thalassiosira spinosa Schrader, Sample 121-758A-19X-CC, high and low focus. 10. Thalassiosira spinosa Schrader?, Sample 121-758A-17X-1, 65-67 cm. 11. Thalassiosira mediaconvexa Schrader, Sample 121-758A-22X-1, 65-67 cm, high and low focus. 12. Thalassiosira spumellaroides Schrader, Sample 121-758A-19X-4, 65-67 cm. 
1
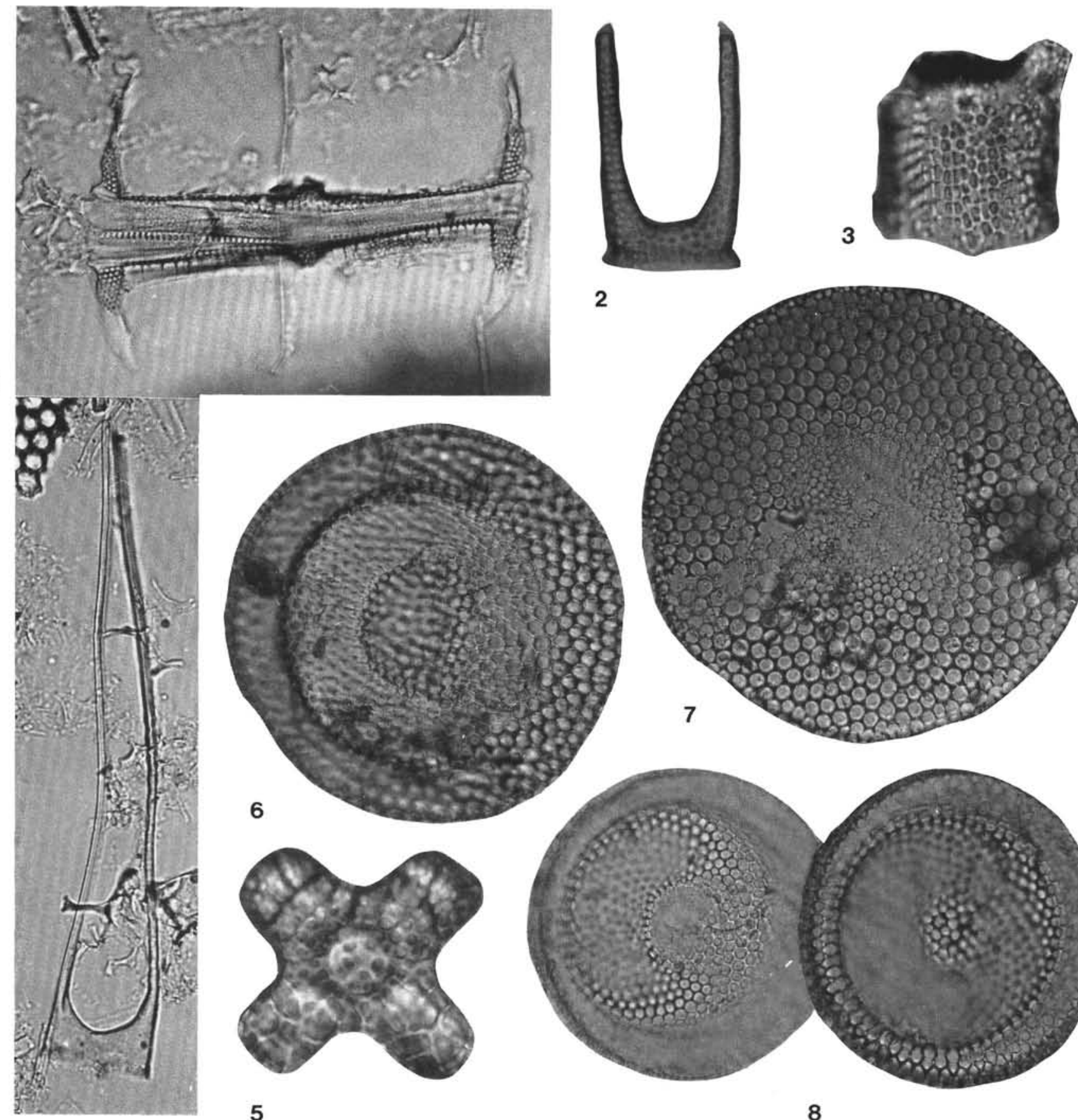

5

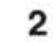

2

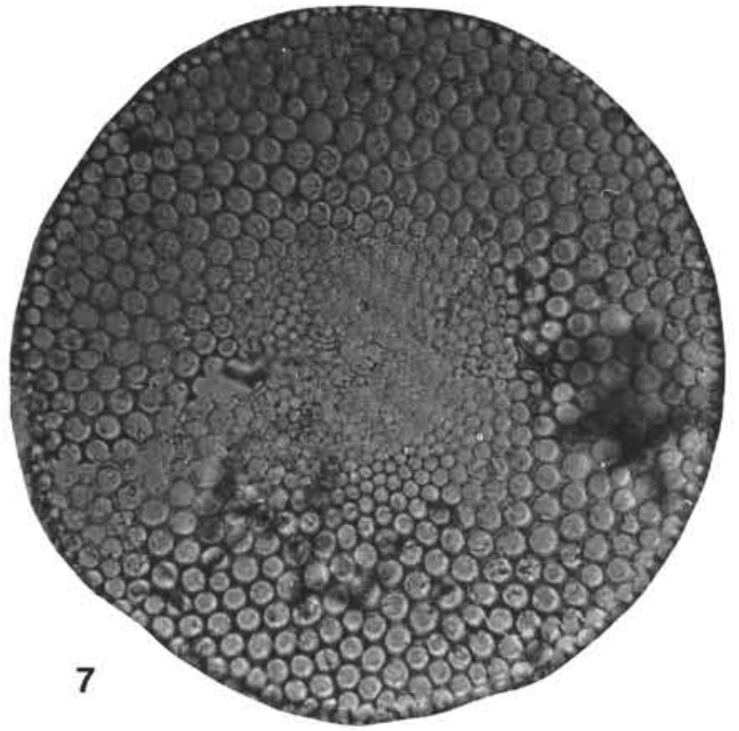

4

Plate 3. Oligocene-Miocene diatoms at Site 758 (scale bar $=25 \mu \mathrm{m}$ for Figs. 6-8; $19 \mu \mathrm{m}$ for Figs. 1, 4; $10 \mu \mathrm{m}$ for Figs. 2, 3, 5). 1. Baxteriopsis brunii (Van Heurck) Karsten, Sample 121-758A-22X-CC (800x). 2. Hemiaulus subacutus Grunow, Sample 121-758A19X-4, 65-67 cm (1500×). 3. Hemiaulus sp. 1, Sample 121-758A-22X-1, 63-65 cm (1500x). 4. Hemiaulus longicornis Greville, Sample 121-758A-21X-CC (800x). 5. Lisitzinia ornata Jousé, Sample 121-758A-22X-1, 63-65 cm (1500x). 6. Craspedodiscus elegans var. asymmetricus n, var. (holotype), Sample 121-758A-19X-4, 65-67 cm (600x). 7. Craspedodiscus barronii Bukry, Sample 121-758A-19X-CC (600×). 8. Craspedodiscus elegans var. asymmetricus Fourtanier n. var., Sample 121-758A$18 \mathrm{X}-\mathrm{CC}$, high and low focus $(600 \mathrm{x})$. 


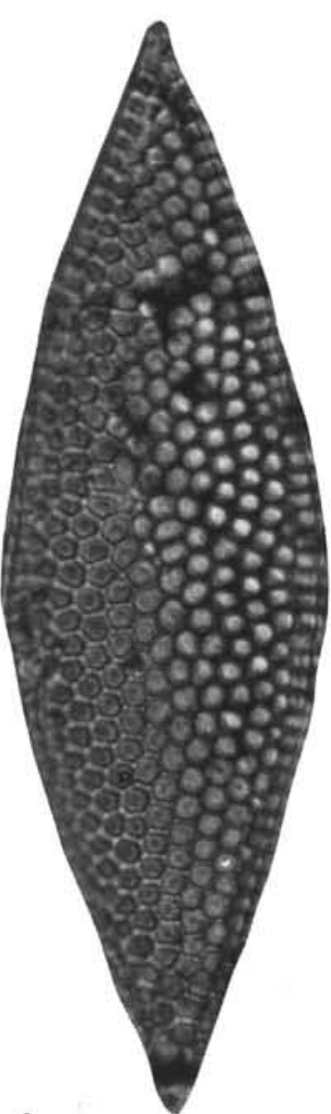

1

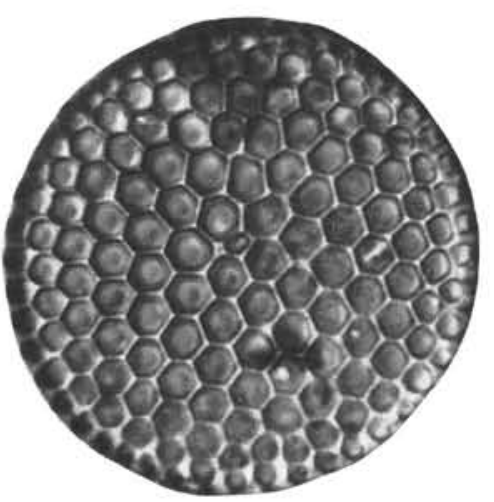

8
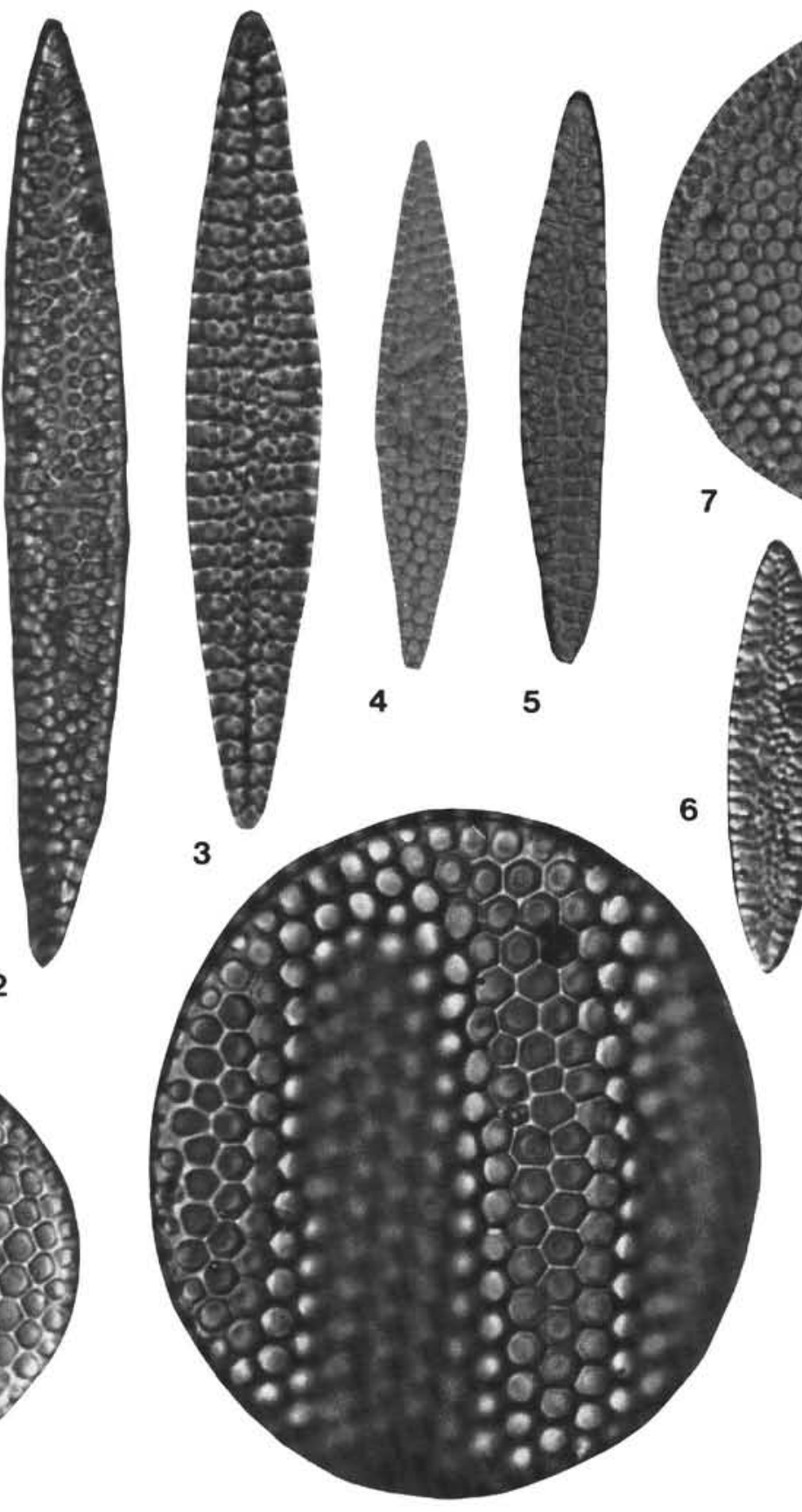

9
6
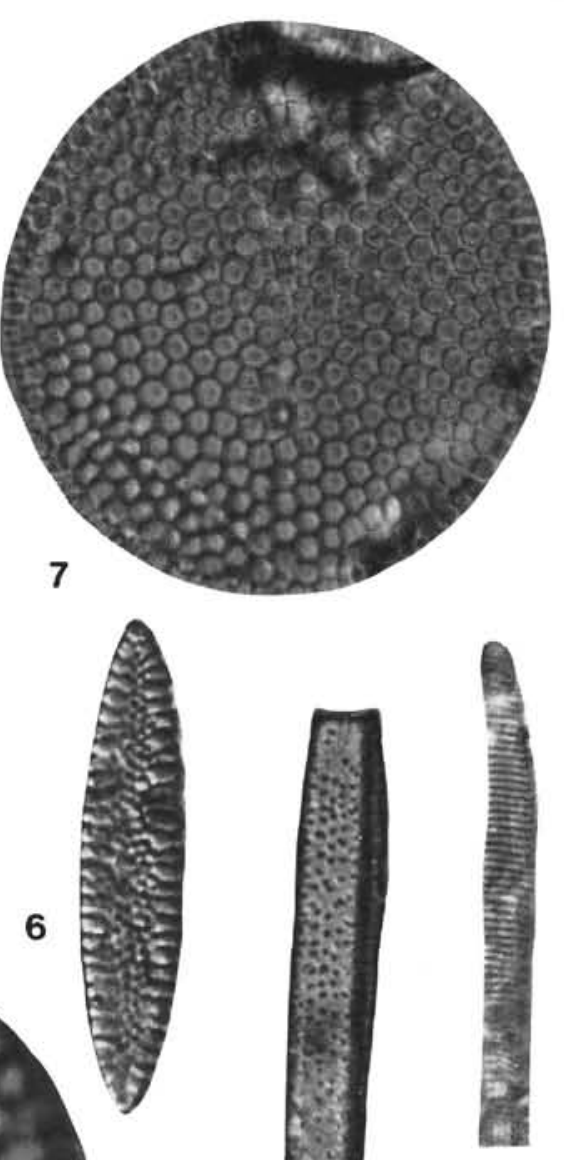

11

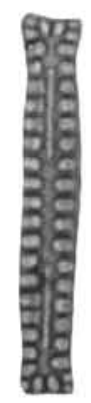

12

Plate 4. Oligocene-Miocene diatoms at Site 758 (all figures $1500 \times$; scale bar $=10 \mu \mathrm{m}$ ). 1. Rossiella magna (Lohman) Fourtanier n. comb., Sample 121-758A-19X-4, 65-67 cm. 2. Rossiella aff. R. symmetrica Fenner (specimen intermediate between Rossiella symmetrica Fenner and Bogorovia veniamini Jousé), Sample 121-758A-21X-3,65-67 cm. 3. Bogorovia veniamini Jousé, Sample 121-758A-22X-1, 63-65 cm. 4. Rossiella paleacea (Grunow) Desikachari et Maheshwari, Sample 121-758A-18X-CC. 5. Rossiella symmetrica Fenner, Sample 121-758A-19X-4, 65-67 cm. 6. Bogorovia sp. 1, Sample 121-758A17X-1, 65-67 cm. 7. Azpeitia oligocenica (Jousé) Sims, Sample 121-758A-22X-1, 63-65 cm. 8. Rocella vigilans Fenner, Sample 121-758A-22X-3, 65-67 $\mathrm{cm}$. 9. Rocella princeps (Jousé) Fenner, Sample 121-758A-21X-5, 65-67 cm. 10. Rhizosolenia aff. alata Brightwell, Sample 121-758A-23X-CC. 11. Nitzschia aff. maleinterpretaria Schrader, Sample 121-758A-16X-CC. 12. Rouxia quadrangula Jousé, Sample 121-758A-19X-CC. 


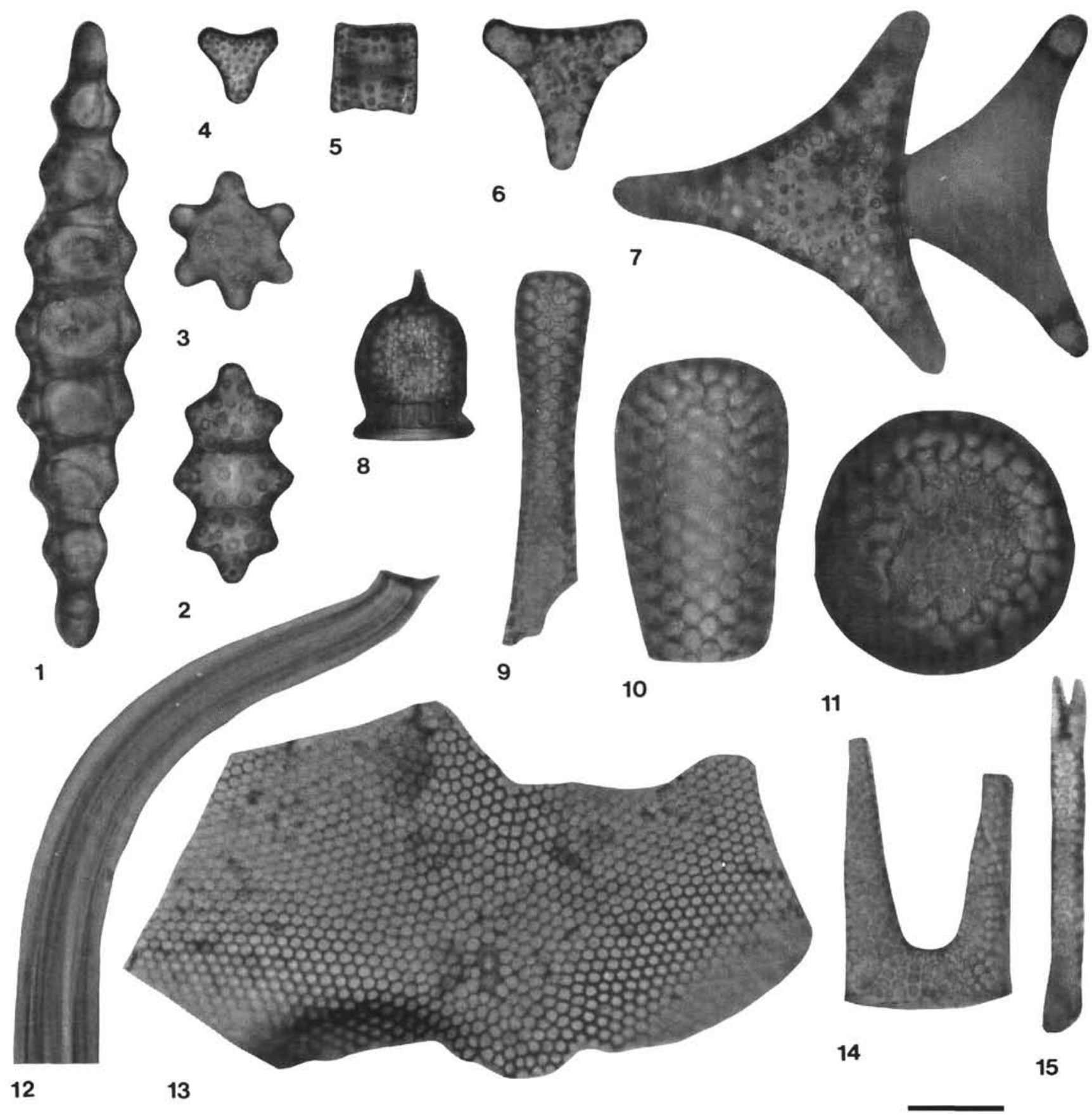

Plate 5. Cretaceous (late Campanian) diatoms at Site 758 (all figures $1500 x$; scale bar $=10 \mu \mathrm{m}$ ). 1, 2. Eunotogramma polymorphus Strelnikova, Sample 121-758A-40X-1, 135-137 cm. 3. Eunotogramma sp. (specimen similar to Eunotogramma polymorphus of Strelnikova, 1974, pl. 52, fig. 8, 9), Sample 121-758A-40X-1, 135-137 cm. 4, 5. Triceratium sp. 1, Sample 121-758A-40X-1, 135-137 cm; (4) valvar view; (5) girdle view. 6. Triceratium schulzii Jousé, Sample 121-758A-40X-2, 106-108 cm. 7. Trinacria pileolus (Ehrenberg) Grunow, Sample 121-758A-40X-1, 135-137 cm, high and low focus. 8. Stephanopyxis schulzii Steinecke, Sample 121-758A-40X-1, 135-137 cm. 9, 10. Gladius speciosus Schulz, Sample 121-758A-40X-1, 135-137 cm. 11. Stephanopyxis reticulata Long, Fuge et Smith, Sample 121-758A-40X-1, 135-137 cm. 12. Rhizosolenia cretacea Hajós et Stradner, Sample 121-758A-40X-1, 135-137 cm. 13. Coscinodiscus morenoensis Hanna, Sample 121-758A-40X-1, 135-137 cm. 14. Hemiaulus sp. 1, Sample 121-758A-40X-1, 135-137 cm. 15. Hemiaulus sp. 2, Sample 121-758A-40X-1, 135-137 cm. 